\title{
Identification of blood vascular endothelial stem cells by the expression of protein $\mathrm{C}$ receptor
}

\author{
Qing Cissy Yu ${ }^{1}$, Wenqian Song ${ }^{1}$, Daisong Wang ${ }^{1}$, Yi Arial Zeng ${ }^{1}$ \\ ${ }^{I}$ The State Key Laboratory of Cell Biology, CAS Center for Excellence in Molecular Cell Science, Institute of Biochemistry and \\ Cell Biology, Shanghai Institutes for Biological Sciences, Chinese Academy of Sciences, Shanghai 200031, China
}

\begin{abstract}
Vascular growth and remodeling are dependent on the generation of new endothelial cells from stem cells and the involvement of perivascular cells to maintain vessel integrity and function. The existence and cellular identity of vascular endothelial stem cells (VESCs) remain unclear. The perivascular pericytes in adult tissues are thought to arise from the recruitment and differentiation of mesenchymal progenitors during early development. In this study, we identified Protein $\mathrm{C}$ receptor-expressing (Procr ${ }^{+}$) endothelial cells as VESCs in multiple tissues. Procr ${ }^{+}$VESCs exhibit robust clonogenicity in culture, high vessel reconstitution efficiency in transplantation, long-term clonal expansion in lineage tracing, and EndMT characteristics. Moreover, Procr $^{+}$VESCs are bipotent, giving rise to de novo formation of endothelial cells and pericytes. This represents a novel origin of pericytes in adult angiogenesis, reshaping our understanding of blood vessel development and homeostatic process. Our study may also provide a more precise therapeutic target to inhibit pathological angiogenesis and tumor growth.
\end{abstract}

Keywords: vascular endothelial stem cell; Procr; lineage tracing; bipotent; mammary gland

Cell Research (2016) 26:1079-1098. doi:10.1038/cr.2016.85; published online 1 July 2016

\section{Introduction}

Vascular growth and remodeling are continuous processes vital for development and homeostasis. Angiogenesis, the process that generates new vessels from pre-existing vessels, persists throughout development, coordinated with organ growth and repair. The walls of stable blood vessels are composed of two distinct cell types: endothelial cells (ECs) and pericytes. ECs form a monolayer of vascular tubes, while pericytes associate with and cover the outside of the endothelial tube. The roles of pericytes in angiogenesis are proven to be critical, including physical vessel stabilization [1], blood flow regulation $[2,3]$ and participation in vascular development and maturation [4-6]. Pericytes are commonly categorized as a subtype of perivascular cells. It is widely recognized that pericytes have mesenchymal or hematopoietic origin and that they are recruited to endothelial

\footnotetext{
Correspondence: Yi Arial Zeng

Tel: +86-21-5492-1433; Fax: +86-21-5492-1225

E-mail: yzeng@sibcb.ac.cn

Received 29 April 2016; revised 20 May 2016; accepted 25 May 2016; published online 1 July 2016
}

tubes [7-11].

ECs are the fundamental building blocks of the vascular architecture. It remains unclear how the EC pool is replenished during homeostasis, i.e., whether it is from the contribution of mature EC proliferation, or the result of vascular stem/progenitor cell activities. The existence and cellular identity of vascular endothelial stem cells (VESCs) also remain controversial. Earlier reports suggest that VESCs originate from circulating bone marrow-derived "endothelial progenitor cells" [13-15]. More recent studies indicate that circulating "endothelial progenitor cells" contribute to vascular endothelium neither in development [16] nor in tumor growth [17]. These studies suggest the existence of resident stem cells in the vascular wall, yet the definitive cellular origin of VESCs for adult angiogenesis has remained unclear. A previous study has implicated $\mathrm{c}-\mathrm{Kit}^{+}$vascular cells as VESCs in the lung using in vitro colony formation and in vivo transplantation assays [18]. It is known that the above assays may stimulate plasticity as cells are taken away from their native habitats [19], thereby genetic fate mapping (in vivo lineage tracing) is needed for the identification of VESCs and interrogation of their properties. It is essential to demonstrate that putative VESCs have 
the ability to give rise to mature endothelial cells both in regeneration and in development, and to establish that putative VESCs encompass two key characteristics that functionally define tissue stem cells: ability to self-renew for a prolonged period, and ability to differentiate into all mature cell types within the tissue [12].

Protein $\mathrm{C}$ receptor (Procr), also known as EPCR, is a single-pass transmembrane protein expressed in ECs, with established roles in anticoagulation and inflammation [20-22]. Procr has also been implicated as a marker for murine hematopoietic stem cells [23], and mammary epithelial stem cells [24]. In this study, we sought to investigate the potential of Procr-expressing ECs with regard to their contribution to blood vessel development and regeneration.

\section{Results}

Procr-expressing EC populations are enriched for stem cells with regenerative capacity

The growth of an organ requires formation of vasculature, which channels vital oxygen, nutrients and defence cells of the immune system. The mammary gland develops mostly in the postnatal stage, which provides an ideal tissue model to study adult angiogenesis. During puberty, the mammary epithelium undergoes robust extension across the fat pad. We observed that this is a process accompanied by extensive vascularization and vessel remodeling. In a 4 -week-old female, the anterior region of the inguinal fat pad is devoid of epithelium (named "empty fat pad") and possesses predominantly capillaries, indicated by the expression of endothelial marker CD31 (PECAM1; Figure 1A). In contrast, at 8 weeks when the epithelium has occupied the fat pad, large blood vessels have also formed in alignment with newly established epithelial branches, with capillaries closely wrapping around the epithelial trunk (Figure 1B). These observations suggest vigorous vascular formation during mammary development, which can be employed to study angiogenesis. We analyzed the location of Procr-expressing cells in the endothelium using immunohistochemical analyses. In the pubertal mammary gland where robust angiogenesis occurs, Procr expression was observed in both tip cells and stalk cells (Figure 1C and 1D); while in the adult mammary gland, Procr $^{+}$cells were predominantly found in stalk cells (Figure 1D). The EC identity of Procr $^{+}$cells was validated by co-staining with EC markers, including Cdh5 (VE-cadherin), CD31 and Emcn (Endomucin; Figure 1D, Supplementary information, Figure S1A and S1B), and with the EC basement membrane proteins laminin and CoIIV (Supplementary information, Figure S1C and S1D). Next, we analyzed the percentage of Procr-expressing cells in ECs using fluorescence-activated cell sorting (FACS). Single cells were isolated from 8-week-old mouse mammary glands, and ECs were detected using blood lineage ${ }^{-}\left(\mathrm{CD} 3 \mathrm{e}^{-}\right.$, $\mathrm{Ly}^{-} \mathrm{G}^{-} / \mathrm{Ly}^{-} \mathrm{C}^{-}, \mathrm{CD} 11 \mathrm{~b}-\mathrm{Gr}^{-}, \mathrm{CD}^{-} 5 \mathrm{R}^{-} / \mathrm{B} 220^{-}$, Ter119), $\mathrm{CD} 105^{+}, \mathrm{CD} 31^{+}$labels [18] (Figure 1E). We found that Procr labels about $4 \%$ of ECs (Figure 1E). Sca $1^{+}$ECs have been reported to enrich for VESCs [25-27]. We found that the majority of ECs are Sca $1^{+}(85.2 \% \pm 3.3 \%$; Supplementary information, Figure S1E) and $\mathrm{Procr}^{+}$ECs are included in the Sca $1^{+}$population (Supplementary information, Figure S1F). It has been reported that VESCs in the lung can be enriched by using the expression of c-Kit [18]. We found that in the mammary vasculature, c-Kit indeed labels a small population of ECs $(2.0 \% \pm$ $0.3 \%$; Supplementary information, Figure S1E). Notably, c-Kit ${ }^{+}$ECs do not overlap with Procr $^{+}$ECs (Supplementary information, Figure S1G), suggesting that these are distinct cell populations.

Next, we assessed the regenerative capacity of Procr ${ }^{+}$ ECs in transplantation assays. Procr ${ }^{+}$and Procr $^{-}$ECs were isolated from adult Actin-GFP mice, mixed with Matrigel and engrafted subcutaneously into recipient nude mice in limiting dilution (illustrated in Figure 1F). At 3 weeks post transplantation, the Matrigel plugs were retrieved and the formation of $\mathrm{GFP}^{+}$blood vessels was scored. We categorized the extent of vessel growth depending on the overall area of the Matrigel plug being occupied. We observed that Procr $^{+}$ECs have drastically higher vessel-forming potential than Procr $^{-}$ECs (Figure 1G). Transplanted Procr $^{-}$ECs infrequently produced partial vessel outgrowth with limited extension (Figure 1G). In our study, c- $\mathrm{Kit}^{+}$cells fall into the Procr $^{-}$population that has low vessel-forming capacity, raising the question whether c-Kit ${ }^{+}$cells are enriched for VESCs in mammary vasculature. To address this, we isolated $\operatorname{Procr}^{+}$ECs and c-Kit ${ }^{+}$ECs from Actin-GFP mammary vasculature and performed subcutaneous transplantation in limiting dilution. Consistent with our earlier results, Procr $^{+}$ECs readily reconstituted new vessels, whereas c-Kit ${ }^{+} \mathrm{ECs}$ had markedly lower in vivo regenerative capacity (Supplementary information, Figure S1H). In colony formation assays described later, Procr $^{+}$ECs formed more colonies compared to $\mathrm{c}-\mathrm{Kit}^{+} \mathrm{ECs}$, while both populations are more clonogenic than the c-Kit ${ }^{-}$, Procr $^{-}$ECs (Supplementary information, Figure S1I).

We noticed that the majority of vessels formed in our transplantation assays and other reports were small vessels resembling the capillary plexus $[18,28]$, probably due to the isolated environment in the Matrigel plug. We sought to evaluate the angiogenic capacity of $\mathrm{Procr}^{+} \mathrm{ECs}$ by transplanting them back to the developing mammary 
A
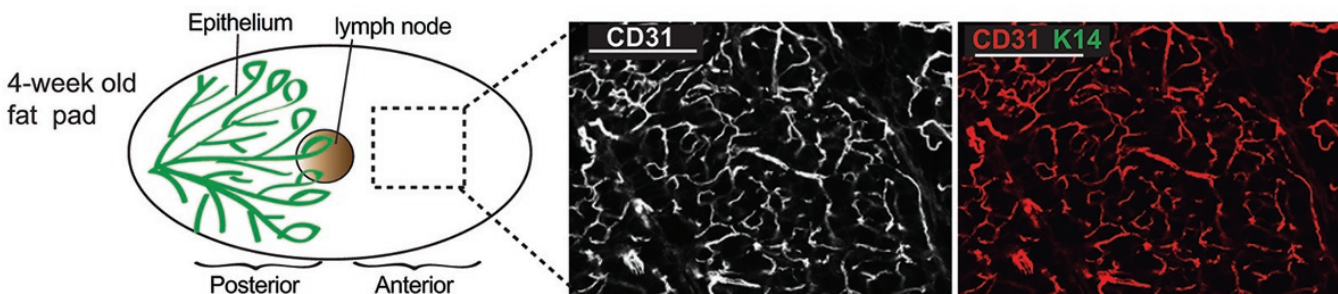

B 8-week old fat pad
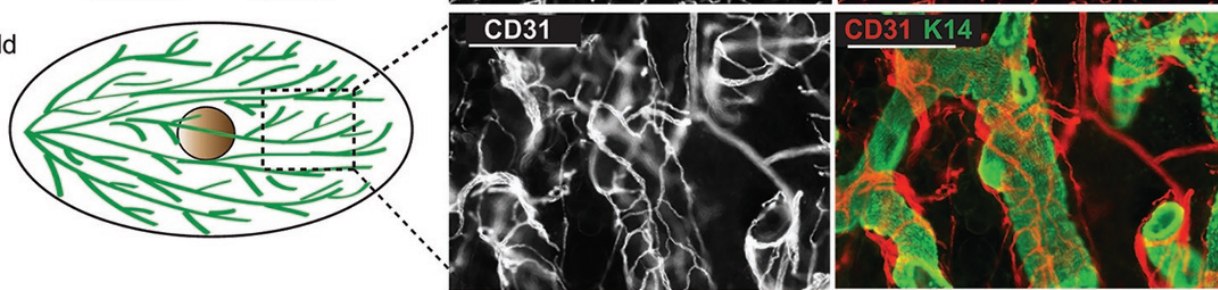

C
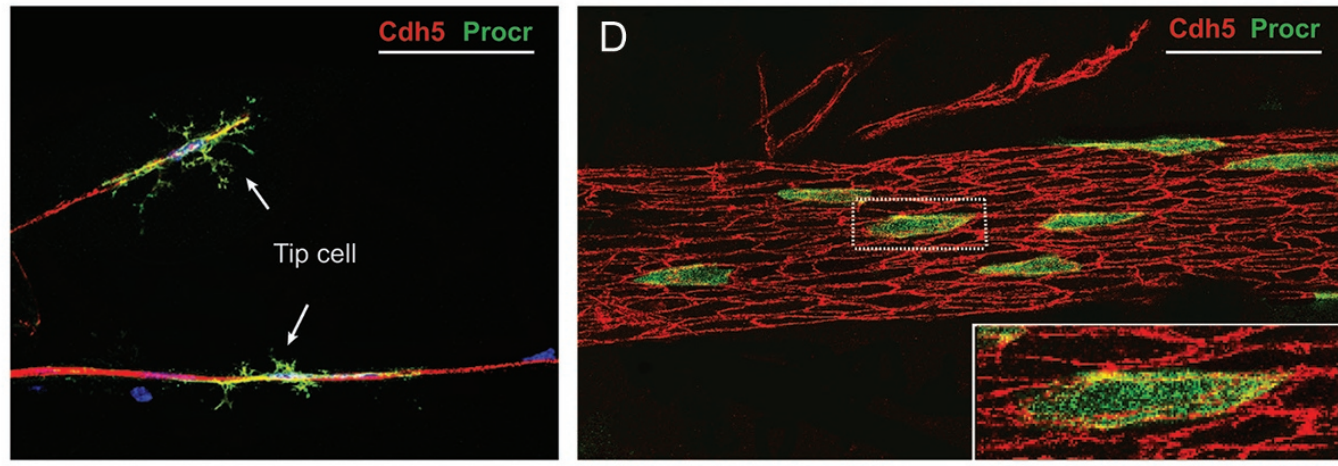

E

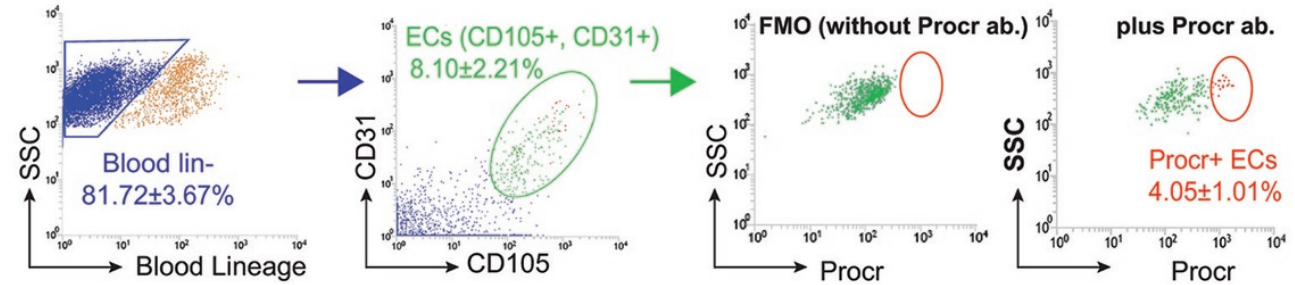

F

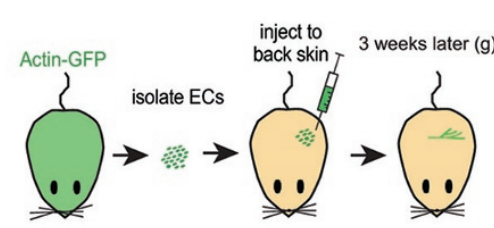

$\mathrm{H}$

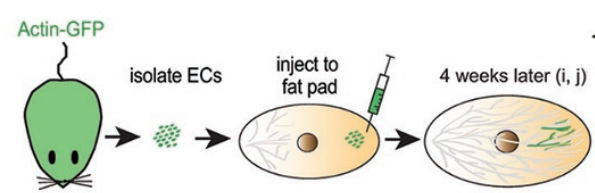

I

\begin{tabular}{lrc}
\multicolumn{1}{c}{ Population } & Cell no. & Outgrowth \\
\hline Lin-,CD105+, & 10,000 & $4 / 4$ \\
CD31+, & 5,000 & $2 / 4$ \\
Procr+ & \\
\hline Lin-,CD105+, & 10,000 & $0 / 3$ \\
CD31+, & 5,000 & $0 / 4$ \\
Procr- &
\end{tabular}

G Population Cell no. Outgrowth Matrigel plug filled

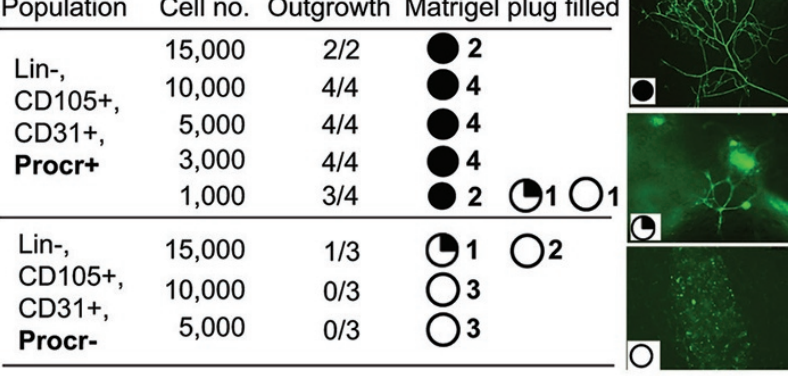

$J$

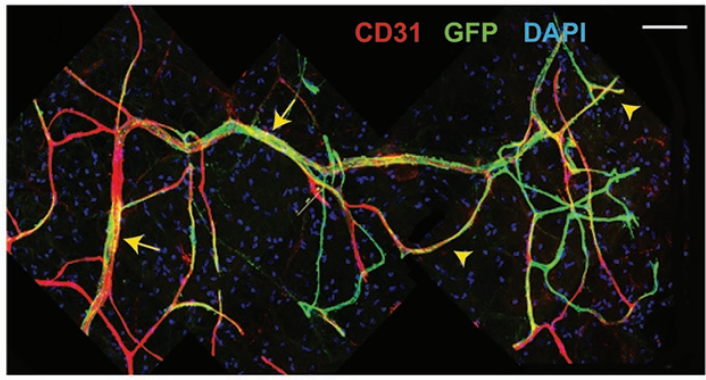


gland. Procr ${ }^{+}$ECs were isolated from 8-week-old $\mathrm{Ac}$ tin-GFP mouse mammary vasculature, injected into the empty fat pad of pubertal recipients (3-week-old), and harvested 4 weeks later (illustrated in Figure 1H). We expected that the robust mammary development during the period (3-7 weeks) could reveal the angiogenic potential of Procr ${ }^{+}$ECs. Indeed, we observed the incorporation of $\mathrm{GFP}^{+}$ECs into large vessels (Figure 1I and 1J, arrows), and also the formation of secondary and tertiary GFP $^{+}$ vessel branches (Figure 1J, arrowheads) in an orderly manner. In contrast, Procr ${ }^{-}$ECs could neither incorporate into the existing vessels nor form new vessels in mammary fat pads (Figure 1I). Together, our data suggest that Procr ${ }^{+}$ECs are enriched for stem cells with robust regenerative capacity.

Procr $^{+}$ECs have robust clonogenicity and their stem cell capacity can be retained in culture

We next examined the behavior of Procr ${ }^{+}$ECs in vitro. We isolated Procr ${ }^{+}$and Procr ${ }^{-}$ECs, and cultured them in a monolayer as previously established [18]. We found that freshly isolated Procr $^{+}$ECs undergo a stable increase in cell number, and can be propagated for at least 10 passages and more than 3 months (Figure 2A). These serially passaged cells largely maintained endothelial properties by showing positive uptake of Acetylated low-density lipoprotein (Ac-LDL), endothelial nitric oxide synthase (eNOS) synthesis, nitric oxide (NO) release and ICAM1 expression upon IL-1 $\beta$ stimulation [29] (Figure 2B and Supplementary information, Figure S2A and S2B). In contrast, Procr ${ }^{-}$ECs had a limited ability to be passaged in culture and deteriorated within 3-4 passages (Figure 2A). Notably, cultured Procr ${ }^{-}$ECs remained Procr ${ }^{-}$, while cultured Procr ${ }^{+}$ECs gave rise to mostly Procr $^{+}$cells and some Procr cells as indicated by FACS analyses (Figure $2 \mathrm{C})$.

Next we examined colony-forming ability using 3D culture. Procr ${ }^{+}$and Procr ${ }^{-}$ECs were FACS-isolated and seeded in 3D methylcellulose culture. We found that Procr $^{+}$ECs have drastically higher clonogenicity compared with Procr ${ }^{-}$ECs (Figure 2D and 2E), and form a "cordlike" structure as previously described [28, 30] (Supplementary information, Figure S2C and S2D). After 14 days in methylcellulose culture, these colonies interconnected and formed a vascular sheet (Supplementary information, Figure S2E). Colonies formed from Procr $^{+}$ECs were dissociated into single cells before being re-plated into fresh methylcellulose. These colonies were capable of generating secondary colonies (Supplementary information, Figure S2F). In contrast, Procr ${ }^{-}$ECs rarely formed colonies in culture and these colonies were smaller in size than Procr $^{+}$colonies and could not be passaged (Figure 2D and 2E). As mentioned above, Pro$\mathrm{cr}^{+}$ECs generated both $\mathrm{Procr}^{+}$and Procr ${ }^{-}$ECs in culture (Figure 2C). These culture-derived Procr $^{-}$ECs lost the colony-forming ability, while their Procr $^{+}$ECs counterpart retained clonogenicity (Figure 2F).

To address whether the cultured Procr $^{+}$ECs retain their stem cell properties, we transplanted the third passage colonies from Procr $^{+}$EC culture into empty fat pads and found that, similar to the freshly isolated primary Procr $^{+}$ECs, they were able to readily reconstitute blood vessels $(8 / 10$, Supplementary information, Figure S2G). To address this at single cell level, we sorted single Procr $^{+}$ECs into 96-well plate pre-coated with methylcellulose. Single $\mathrm{GFP}^{+}$colonies formed from single Procr $^{+}$EC were picked and individually transplanted subcutaneously into empty fat pads (illustrated in Figure $2 \mathrm{G})$. $\mathrm{GFP}^{+}$outgrowths were readily detected in both back skin $(9 / 10)$ and mammary fat pad $(8 / 10$; Figure $2 \mathrm{H}$ and 2I). The endothelial identity of these $\mathrm{GFP}^{+}$vessels was validated by $\mathrm{CD} 31$ staining (Figure $2 \mathrm{H}$ and $2 \mathrm{I}$ ). To investigate whether the $\mathrm{GFP}^{+}$vessels in mammary fat pads are part of functional circulatory vasculature, isolectin was administered by intravenous injection prior to harvest [31]. We observed that the $\mathrm{GFP}^{+}$vessels were isolectin ${ }^{+}$,

Figure 1 Procr-expressing endothelial cells are enriched for stem cells with regenerative capacity. (A) Illustration of pubertal mammary gland (4-week-old) and staining of endothelium (CD31) and epithelium (outlined by K14) in empty fat pad section prior to epithelial occupancy. Scale bar, $150 \mu \mathrm{m}$. (B) Illustration of adult mammary gland (8-week-old) and staining of blood vessel (CD31) and epithelium (K14) in section of fat pads with epithelium penetration. Scale bar, $150 \mu \mathrm{m}$. (C, D) Immunohistochemistry indicating the expression of Procr in both tip cells (C) and stalk cells (D). Scale bar, $50 \mu \mathrm{m}$. (E) FACS analysis of Procr expression in 8-week-old C57BL/6 mammary gland ECs. (F, G) Schematic illustration of subcutaneous transplantation assays. Procr ${ }^{+}$ECs and Procr ${ }^{-}$ECs were isolated from 8-week-old Actin-GFP mammary glands by FACS. The isolated ECs with GFP label were transplanted in limiting dilution into recipients under the flank skin (subcutaneous) as indicated (F). The degree of endothelial vessel outgrowth was evaluated based on the occupancy of Matrigel plug. The representative images were shown on the right. Data were pooled from five independent experiments (G). (H-J) Transplantation of sorted Procr ${ }^{+}$vs Procr ${ }^{-}$ECs in limiting dilution into the empty fat pad of 3-week-old pubertal mammary gland $\mathbf{( H )}$. Fat pads of recipients were harvested and the $\mathrm{GFP}^{+}$vessel outgrowths were analyzed at 4 weeks post transplantation (I). Whole-mount confocal image indicating the integration and contribution of transplanted $\operatorname{Procr}^{+}$ECs $\left(\mathrm{GFP}^{+}\right)$to host mammary vasculature. Endothelial cells were counterstained with CD31 (J). Scale bar, $50 \mu \mathrm{m}$. 
A

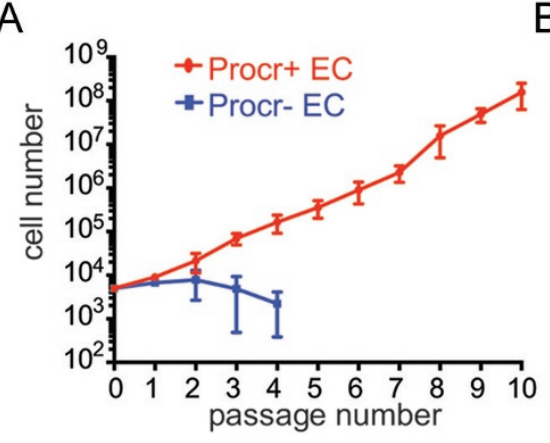

C

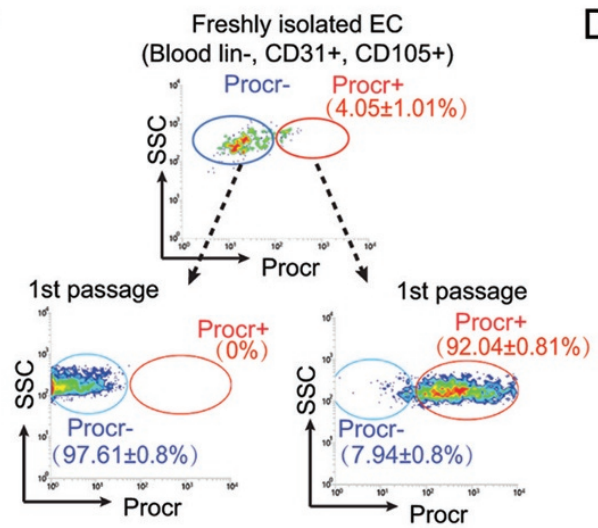

D

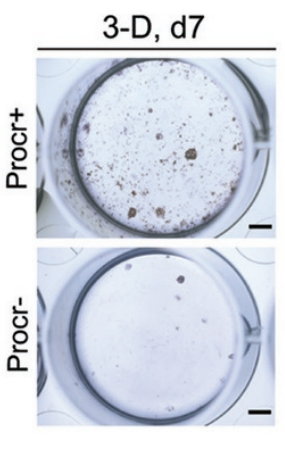

G

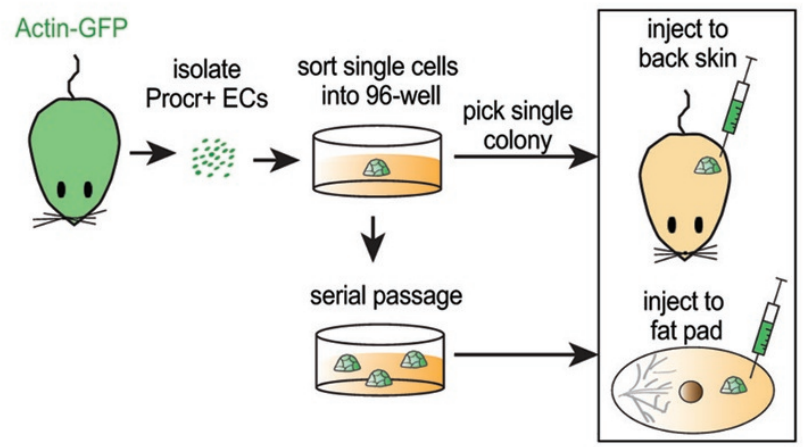

2-D, 5th passage, Procr+ EC

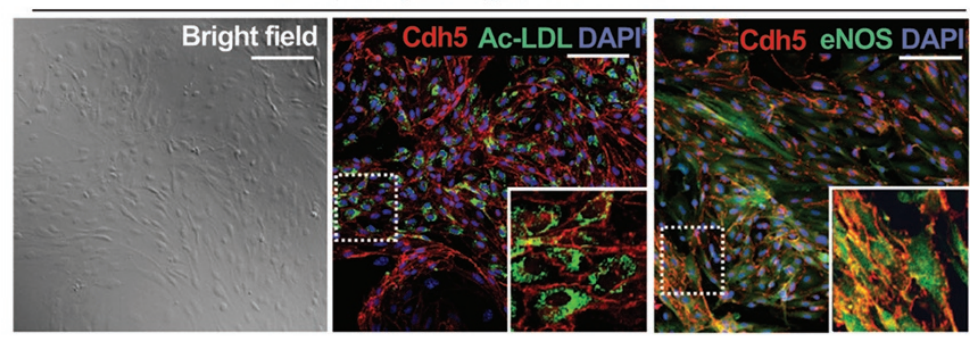

E

$\mathrm{F}$

Freshly isolated EC
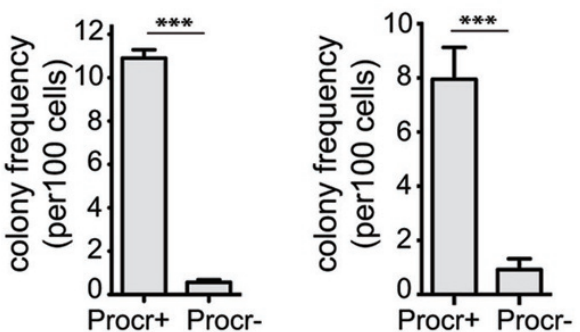

$\mathrm{H}$

Single colony (1st passage) subcutaneous transplantation (outgrowth 9 out of 10)

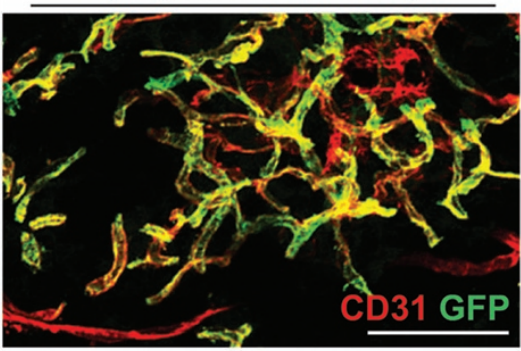

Single colony (1st passage) fat pad transplantation (outgrowth 8 out of 10)
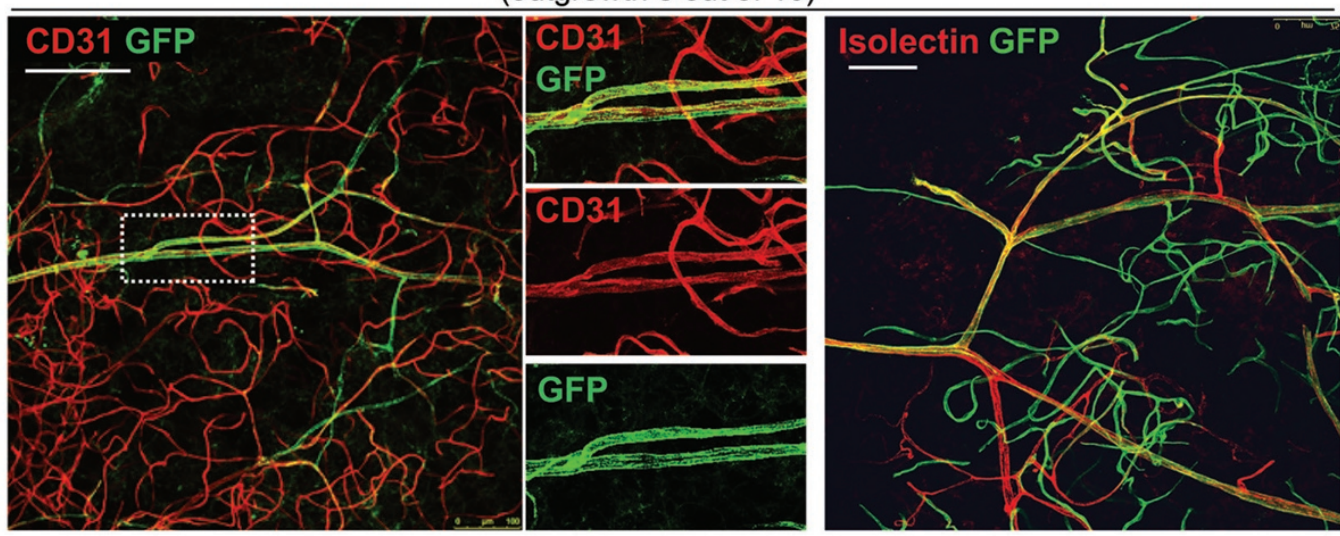
indicating that they are luminized and connected with the host vasculature (Figure 2I). As mentioned above, Procr $^{+}$ ECs generated both Procr $^{+}$and Procr $^{-}$ECs in culture. To further discern the contribution of $\mathrm{Procr}^{+}$and Procr ${ }^{-}$cells upon transplantation, Procr $^{+}$and Procr ${ }^{-}$cells were isolated from the Procr ${ }^{+}$EC culture and transplanted (Supplementary information, Figure S2H). We observed that these culture-derived Procr ${ }^{-}$cells lost the regenerative capacity, while their Procr $^{+}$counterparts could still readily generate new vessels (Supplementary information, Figure S2H). These results suggest that Procr $^{+}$ECs can generate $\mathrm{Procr}^{+}$descendants that retain stem cell properties following cell culture.

In addition to the mammary vasculature, we also examined the behavior of Procr ${ }^{+}$ECs in the skin. A similar percentage of Procr $^{+}$ECs $(8.8 \% \pm 0.4 \%)$ was observed following FACS analysis of abdominal skin endothelium (Supplementary information, Figure S3A). Isolated Procr $^{+}$ECs from the skin were able to expand in 2D culture and they maintained their endothelial properties as shown by Ac-LDL uptake, NO release and ICAM1 expression upon stimulation, whereas Procr $^{-}$ECs had limited growth in culture (Supplementary information, Figure S3B-S3E). Procr ${ }^{+}$ECs from the skin also had significantly higher colony formation ability in 3D culture (Supplementary information, Figure S3F), as well as in vivo vessel formation ability compared with Procr $^{-}$ECs (Supplementary information, Figure S3G). Together, these results suggest that skin and mammary gland Pro$\mathrm{cr}^{+}$ECs are enriched for stem cells with robust colony and blood vessel formation abilities.

Procr $^{+}$ECs contribute to EC expansion in lineage tracing

Next we examined the contribution of Procr $^{+}$ECs un- der physiological conditions by in vivo genetic fate mapping. We used a Procr ${ }^{\text {CreERT2-IRES-tdTomato/t }}$ knock-in mouse model, in which a CreERT2-IRES-tdTomato cassette is inserted after the first ATG codon of Procr [24]. By crossing the Procr ${ }_{\text {CreERT2-IRES-tdTomato/+ }}$ allele (Procr ${ }^{\text {CreERT2 } /+^{+}}$) with the Rosa26 $6^{m T m G /+}\left(R 26^{m T m G /+}\right)$ reporter strain [32], we were able to trace the fate of endogenous Procr ${ }^{+}$ECs in vivo by GFP expression (Figure $3 \mathrm{~A}$ ). We first tracked the developmental fate of Procr $^{+}$ECs by administering tamoxifen (TAM) to Procr ${ }^{\mathrm{CreERT2/+}} ; R_{2} 6^{m T m G /+}$ pubertal mice (5-week-old) and analyzing the labeled cells both at short term (2 days) and at extended time periods (up to 10 months; Figure 3B). Expression of GFP was not detected in un-induced mice (data not shown). Short-term tracing analyses ( 2 days) using confocal whole-mount imaging enabled visualization of single GFP-labeled cells on pre-existing blood vessels (Figure 3C and 3D). After 7 and 14 days of tracing, the $\mathrm{GFP}^{+}$cells expanded in number (Figure 3E-3H). We continued to observe clonal expansion after 2 months of tracing (Figure 3I). Cdh5 and laminin staining validated the endothelial identity of $\mathrm{GFP}^{+}$cells (Figure 3J and 3K). Quantifying the number of cells in each clone at each analysis time point, ranging from 1 cell at 2 days to about 12 cells at 2 months, further demonstrated the expansion of clone sizes during development (Figure 3L). When the tracing was extended to 10 months, we frequently found that large $\mathrm{GFP}^{+}$clones constituted entire vessels (Figure $3 \mathrm{M}$ and $3 \mathrm{~N}$ ). At this stage it is likely that some neighboring clones have merged, interfering with a precise quantification of cells within individual clones. Together, these data suggest that Procr ${ }^{+}$ECs are stem cells that contribute to EC expansion in normal development.

Of note, at the start of TAM induction (5-week), the forefront of the epithelium was at a position slightly past

Figure 2 Procr $^{+}$ECs have robust clonogenicity and their cultured progenies retain stem cell capacities. (A, B) Procr ${ }^{+}$ECs and Procr $^{-}$ECs were sorted from 8-week-old mammary fat pads and plated in 2-D culture in equal amount. Cell numbers at each passage were quantified and displayed as mean \pm SD. Data are pooled from 6 independent experiments (A). Cultured Procr ${ }^{+}$ ECs (left, bright field) showed positive uptake of Ac-LDL (middle) and positive staining for eNOS Ser1117 (right, B). Scale bar, $100 \mu \mathrm{m}$. (C) Cultured Procr ${ }^{+}$ECs or Procr ${ }^{-}$ECs from the first passage were subjected to FACS analysis for the expression of Procr. Procr ${ }^{-}$ECs remained negative for Procr expression in culture. Procr ${ }^{+}$ECs gave rise to majority of Procr $^{+}$cells and a small percentage of Procr $^{-}$cells as indicated. (D) Sorted Procr ${ }^{+}$ECs or Procr ECs from 8-week-old CD1 mammary vasculature were cultured in semi-solid 3D methylcellulose medium. Representative pictures of formed colonies on day 7 were shown. Scale bar, $1 \mathrm{~mm}$. (E, F) Quantification of colony forming frequencies of Procr $^{+} \mathrm{EC}$ or Procr ${ }^{-}$EC freshly isolated from the mammary gland $(E)$, and from cultured Procr ${ }^{+}$ECs (F). Colony numbers were counted on day 7 . Data are pooled from 3 independent experiments and displayed as mean $\pm \mathrm{SD}$. ${ }^{* *} P<0.01$. (G) Schematic illustration of transplantation assays using colonies derived from single Procr ${ }^{+}$ECs. (H) Single colonies derived from single GFP-labeled Procr ${ }^{+}$ECs were mixed with Matrigel and subcutaneously transplanted to recipients. The outgrowths were analyzed at 3 weeks post injection by wholemount staining with EC marker CD31. GFP ${ }^{+}$endothelia were detected in 9 out of 10 Matrigel plugs. Scale bar, $50 \mu \mathrm{m}$. (I) Single colonies derived from single GFP-labeled Procr $^{+}$ECs were transplanted to empty mammary fat pads. The outgrowths were analyzed at 4 weeks post injection by whole-mount staining with EC marker CD31 (left). GFP ${ }^{+}$endothelia were detected in 8 out of 10 fat pads. Intravenous injection in recipient mice with isolectin and the following staining indicated that the outgrowths have formed luminized vessels (right). Scale bar, $50 \mu \mathrm{m}$. 
A Procr-CreERT2;R26-mTmG B
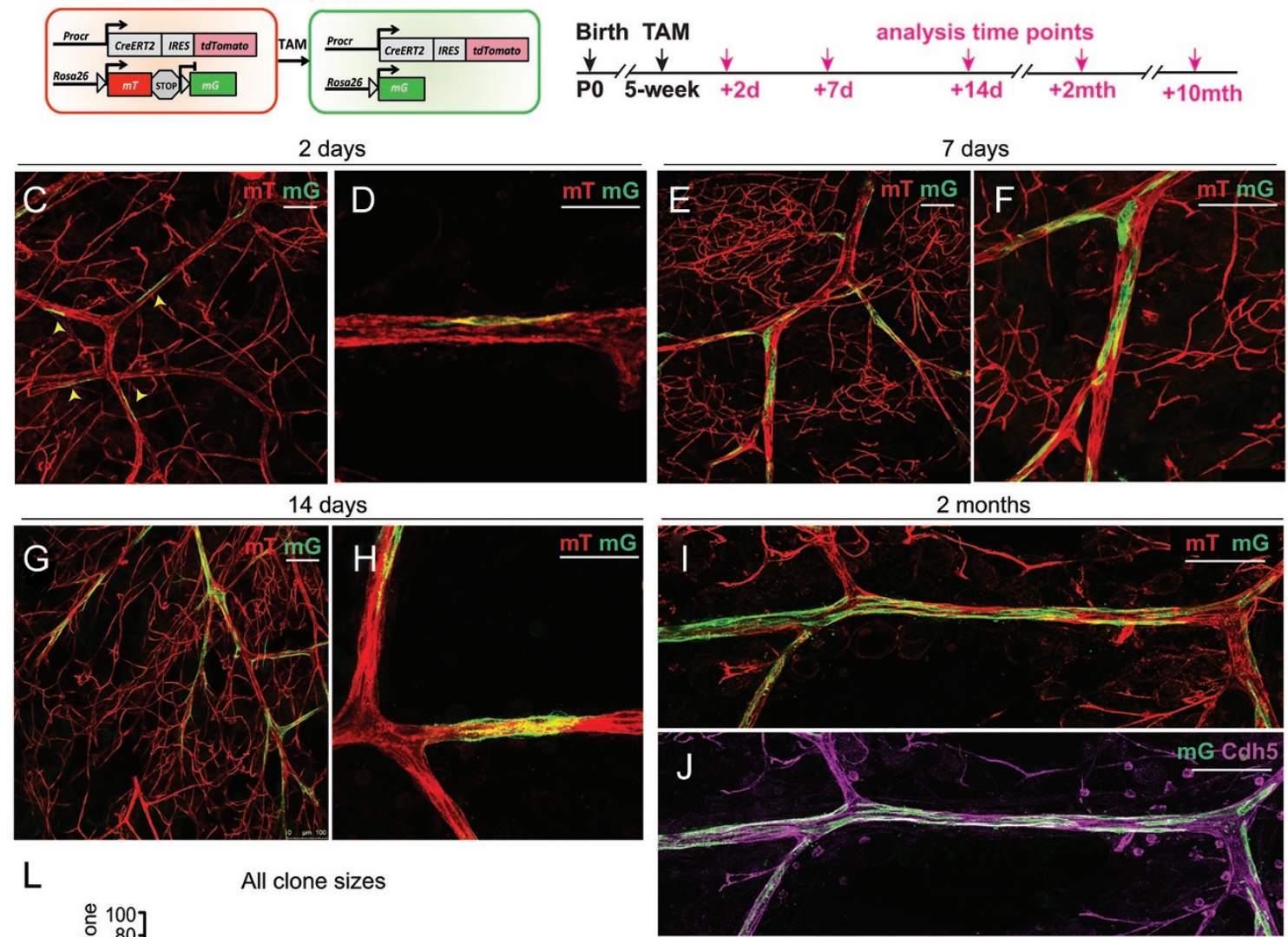

$\mathrm{L}$
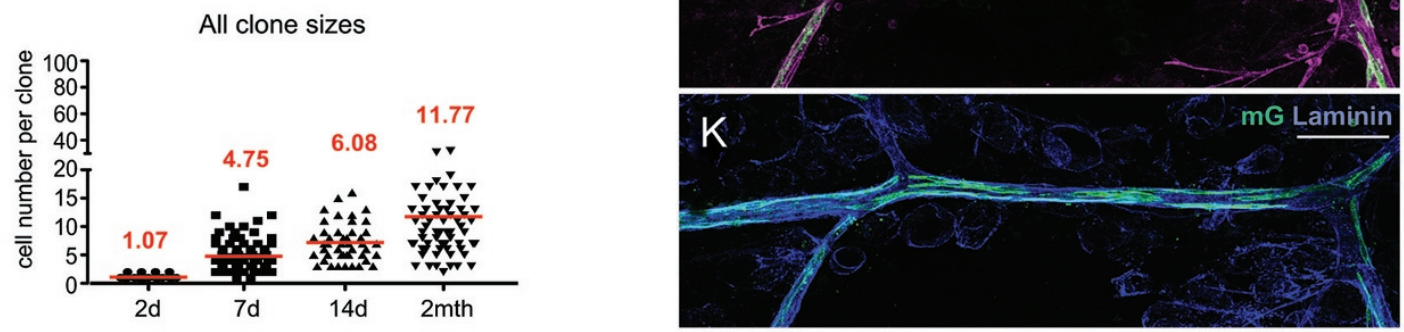

10 months
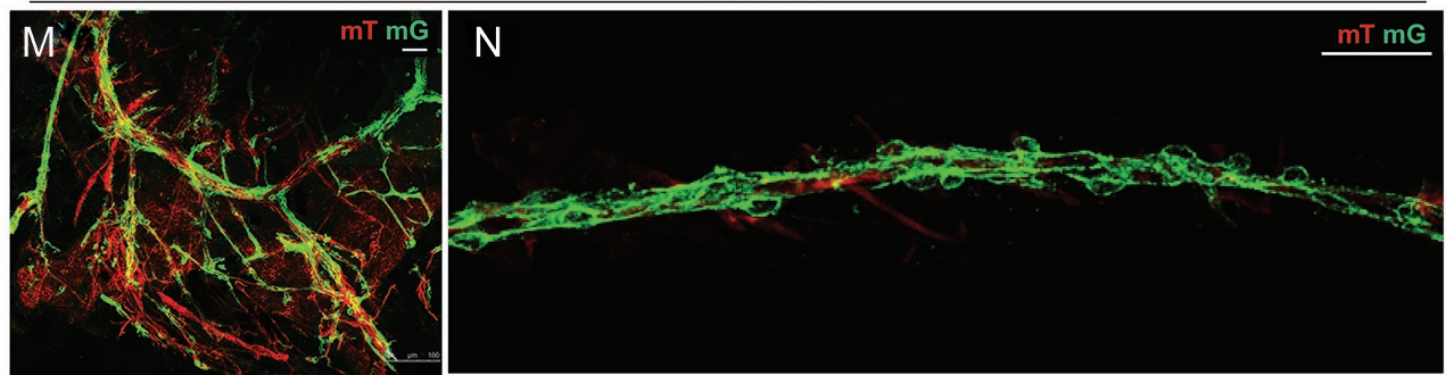

Figure 3 Lineage tracing of Procr ${ }^{+}$ECs demonstrates their contribution to EC clonal expansion during development. (A) Illustration of lineage tracing strategy using Procr ${ }^{\mathrm{CreERT2/+}} ; R 26^{\mathrm{mTmG/+}}$ line. (B) Experimental setup used in short-term (2 days) and long-term tracing (7 days to 10 months) as indicated. (C, D) Whole-mount confocal imaging of mammary vasculature at 2 days post TAM administration showing individual GFP ${ }^{+}$ECs on existing vasculature with zoom out (C) and zoom in (D) views. Scale bar, $50 \mu \mathrm{m}$. (E-H) Whole-mount confocal imaging of mammary vasculature at 7 days (E, F) and 14 days (G, H) post TAM administration indicating clonal expansion of GFP ${ }^{+}$cells. Scale bar, $50 \mu \mathrm{m}$. (I-K) Whole-mount confocal imaging of mammary vasculature at 2 months post TAM administration. Staining of Cdh5 (J) and laminin (K) confirmed the EC identity of GFP $^{+}$clones. Scale bar, $50 \mu \mathrm{m}$. (L) Quantification of cell numbers per GFP ${ }^{+}$clone indicating the expansion of clone sizes at 2 days, 7 days, 14 days and 2 months post TAM treatment. Data were pooled from at least 3 mice for each tracing time point and are presented as mean \pm SD. $(\mathbf{M}, \mathbf{N})$ Whole-mount confocal imaging of mammary vasculature at 10 months post TAM administration. 
the lymph node; after 14 days of tracing, the forefront of the epithelium has finished its extension, reaching the other end of the fat pad (illustrated in Figure 1A and 1B, and Supplementary information, Figure S4A). Given the results in Figure 1A and 1B, at this stage the anterior compartment hosts more newly formed vasculature, while the region posterior to the lymph node represents less dynamic, more established vessel development. We quantified the sizes of $\mathrm{GPF}^{+}$clones in these two compartments, and observed that anterior clones $(6.44 \% \pm 0.38 \%$ cells per clone) are larger than the posterior clones $(3.79 \%$ $\pm 0.27 \%$ cells per clone; Supplementary information, Figure S4B), in line with the idea that angiogenesis is more robust in the anterior region.

Next we examined the contribution of Procr $^{+}$ECs by carrying out lineage tracing in 8-week-old adult mice (Supplementary information, Figure S4C). Consistently, we observed a significant increase in clone size between tracing done for 2 days and for 2 months (Supplementary information, Figure S4D-S4F). Notably, the $\mathrm{GFP}^{+}$clone sizes induced from adult tracing were relatively smaller compared with the ones induced in pubertal tracings ( 8.8 cells per clone in adult tracing at 2 months compared to 11.8 cells per clone in pubertal tracing at 2 months; Supplementary information, Figure S4D and Figure 3L), consistent with the notion of slower vascular turnover in the adult mammary gland.

\section{Lineage tracing reveals that $\mathrm{Procr}^{+}$ECs can give rise to pericytes}

When analyzing $\mathrm{GFP}^{+}$clones in the previous tracing experiments, to our surprise, we observed the presence of $\mathrm{GFP}^{+}$pericytes (see below), suggesting that either Procr also labels pericytes, or that Procr $^{+}$ECs give rise to new pericytes during the tracing period. Thus we investigated whether Procr $^{+}$cells express the pericyte marker nerve/ glial antigen-2 (NG2) [33]. FACS analyses demonstrated that neither all ECs (grey, blood $\operatorname{lin}^{-}, \mathrm{CD} 31^{+}, \mathrm{CD} 105^{+}$) nor Procr ${ }^{+}$ECs (red, blood lin ${ }^{-}, \mathrm{CD} 31^{+}, \mathrm{CD} 105^{+}$, Procr ${ }^{+}$) express the pericyte marker NG2 (Figure 4A). Co-staining of Procr and NG2 further supported the conclusion; no overlap of $\operatorname{Procr}^{+}$ECs with $\mathrm{NG}^{+}$pericytes was detected (Figure 4B, $n>200$ Procr $^{+}$ECs scored). Of note, pericytes do not express CD31 and CD105, and should appear in the non-EC fraction that includes stromal cells. Since Procr labels some stromal cells [24], we next examined the Procr $^{+}$cells in relation to pericytes in the non-EC compartment (blood lin ${ }^{-}, \mathrm{CD}^{-} 1^{-}, \mathrm{CD} 105^{-}$). FACS analyses indicated that Procr ${ }^{+}$cells and pericytes $\left(\mathrm{NG}^{+}, \mathrm{Pdgfrb}^{+}\right)$[34] are two distinct populations with no overlap in this compartment (Figure 4C), suggesting that pericytes do not express Procr.
We next investigated whether Procr $^{+}$ECs give rise to new pericytes using lineage tracing. First, we used Procr $^{\mathrm{CreERT2/+}} ; R 26^{m T m G /+}$ mice and initiated the tracing by TAM administration at 5 weeks. We examined the Pro$\mathrm{cr}^{+}$cell distribution in mammary vasculature over time. At 2 days post TAM induction, consistent with previous results (Figure 3C and 3D), the majority of GFP-labeled ECs remained as single-cell clones $(94.5 \%$ out of $>200$ $\mathrm{GFP}^{+}$clones scored, $n=6$ mammary glands from 3 independent experiments). All $\mathrm{GFP}^{+}$cells are $\mathrm{CD} 31^{+} \mathrm{ECs}$ and do not express the pericyte marker NG2 (Figure 4D). In $\mathrm{PrOCr}^{\mathrm{CreERT2/+}} ; \mathrm{R}^{2} 6^{\mathrm{mTmG} /+}$ mammary glands examined 2 months after induction of tracing, we observed the presence of $\mathrm{GFP}^{+}$pericytes within $\mathrm{GFP}^{+}$clones using the pericyte marker NG2 (arrowheads in Figure 4E and Supplementary information, Figure S5A). The appearance of $\mathrm{GFP}^{+}$pericytes was further confirmed by using additional pericyte markers, Desmin [35] (Figure 4F), $\alpha$ SMA [36] (Supplementary information, Figure S5B) and Pdgfrb (Supplementary information, Figure S5C). Moreover, laminin staining indicated that the $\mathrm{GFP}^{+}$pericytes and the $\mathrm{GFP}^{+} \mathrm{ECs}$ share the same basement membrane, further validating the pericyte identity of the $\mathrm{GFP}^{+}, \alpha \mathrm{SMA}^{+}$cells (Supplementary information, Figure S5D). Quantification of $\mathrm{GPF}^{+}$pericytes seen in $\mathrm{GFP}^{+}$clones in each analysis time point indicated that the frequency of pericyte(s) labeling increased over time, from $0 \%$ at 2 days to $16.1 \%$ at 2 months to $32.1 \%$ at 10 months (Figure $4 \mathrm{G}$ ). Notably, we were only able to detect $\mathrm{GFP}^{+}$pericytes on smaller vessels. We speculated that it could be because large vessels have functionally matured prior to the initiation of tracing, thereby no pericyte differentiation occurs during the tracing period. FACS analyses further supported the appearance of $\mathrm{GFP}^{+}$pericytes and their increasing number over time. We first quantified the percentage of GFP ${ }^{+}$ ECs, which allowed us to determine the overall labeling efficiency and expansion of clones in these experiments. The percentage of $\mathrm{GFP}^{+}$ECs started from $0.86 \%$, and increased to $8.51 \%$ at 2 months and $23.49 \%$ at 10 months (Figure 4H and Supplementary information, Figure S6A). This is consistent with the increasing clone sizes described above (Figure 3L). Quantification of pericytes by FACS demonstrated increasing numbers of GFP pericytes during the tracing time, from $0 \%$ at 2 days to $2.98 \%$ at 2 months to $7.06 \%$ at 10 months (Figure $4 \mathrm{I}$ and Supplementary information, Figure S6B). Together, these results suggest that more pericytes had been generated from Procr $^{+}$ECs over time.

To further validate the Procr $^{+}$EC origin of pericytes, we used the multicolor $R 26 R$-Confetti $\left(R 26^{\text {Confetti }}\right)$ reporter [37]. TAM injection in $\mathrm{Procr}^{\mathrm{CreERT2/+}} ; R 26^{\mathrm{Confetti/} /}$ mice allows Procr ${ }^{+}$cells to randomly adopt one of the four flu- 
A

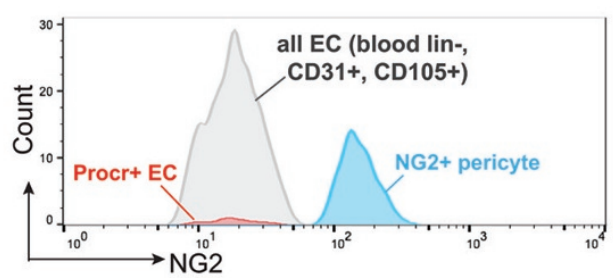

C

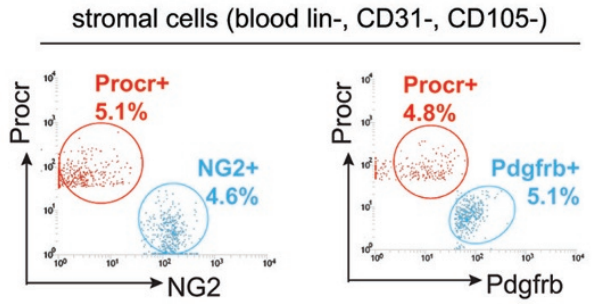

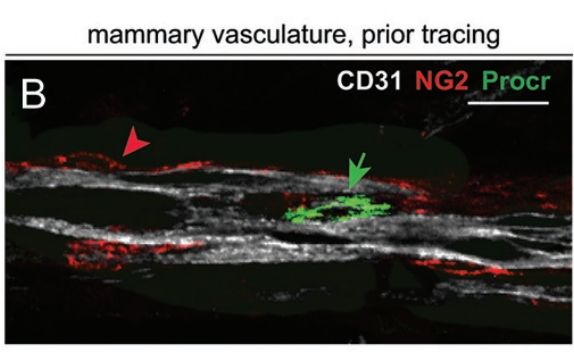

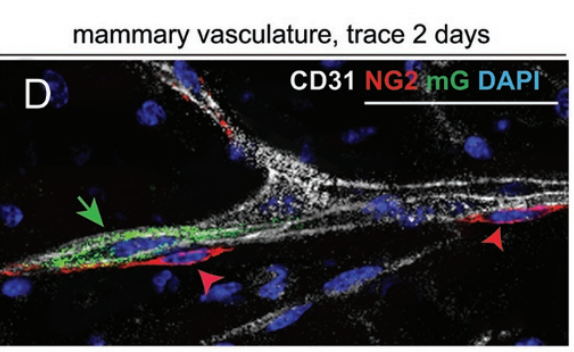

mammary vasculature, trace 2 months

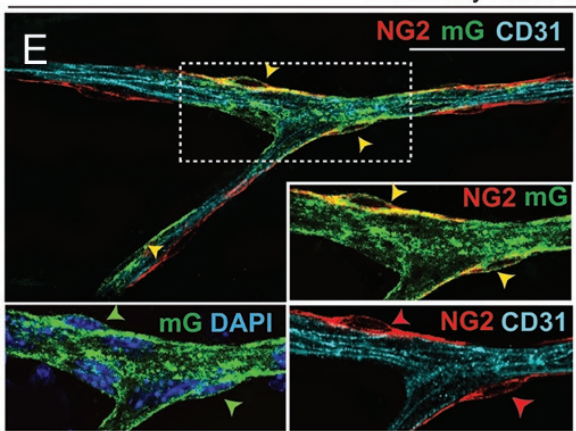

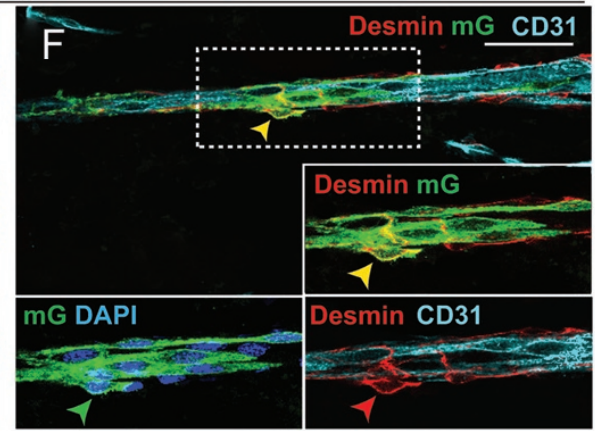
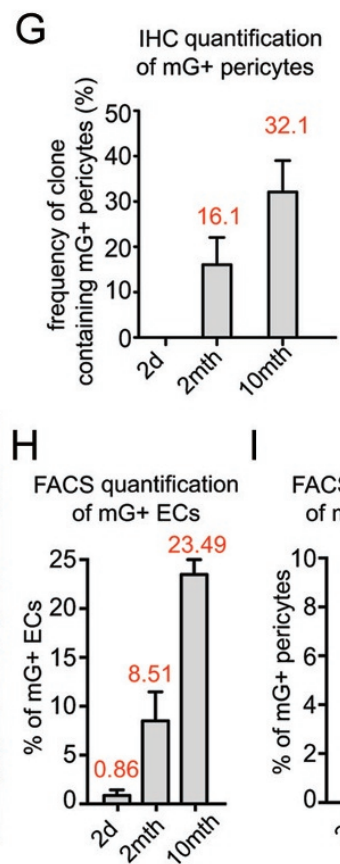

I

FACS quantification of $\mathrm{mG}+$ pericytes

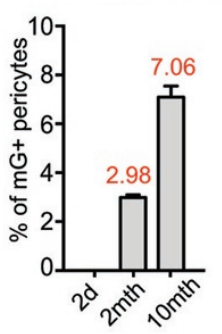

Procr-CreERT2;R26-Confetti, trace 1-2 months
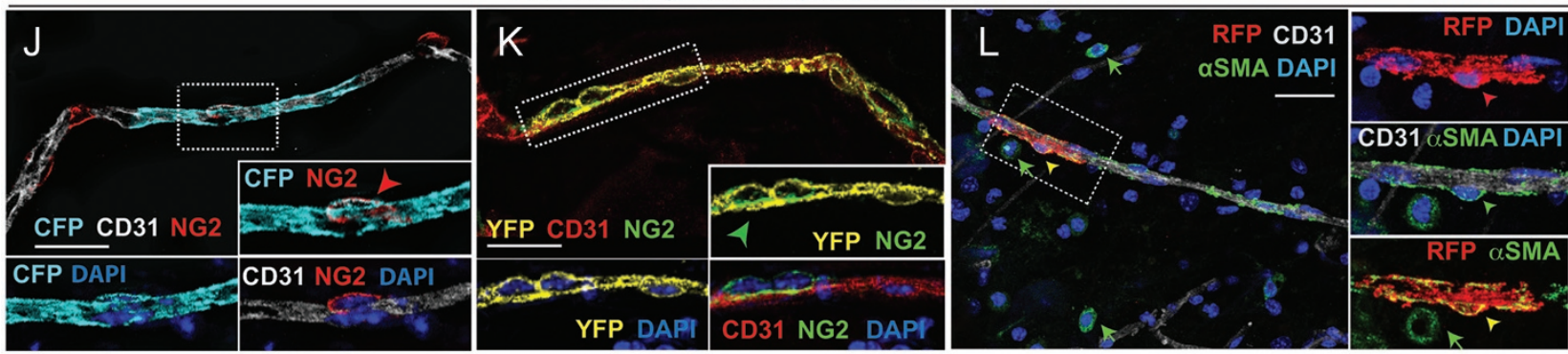

\section{M}

Cdh5-CreERT2;R26-mTmG

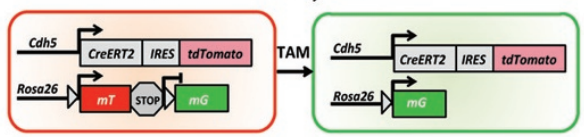

Birth TAM analysis time point

$\frac{\downarrow}{\text { P0 }} / \frac{\downarrow}{\text { 5-week }}+4$ weeks

Cdh5-CreERT2;R26-mTmG, trace 1 month

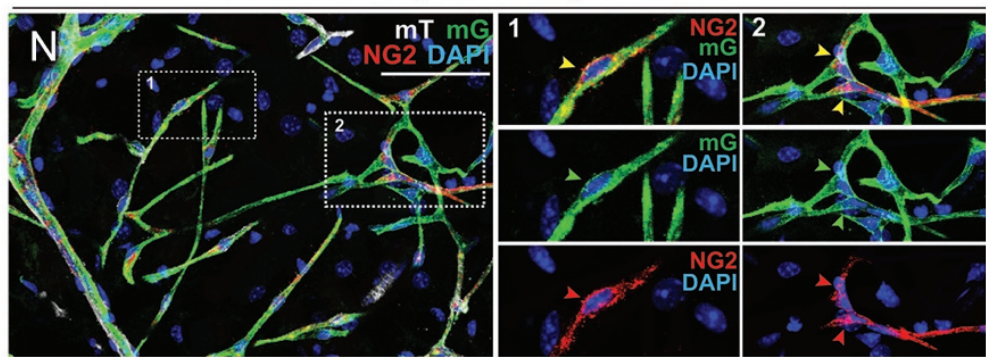


orescent colors. We initiated the tracing at 5 weeks and analyzed the contribution of Procr ${ }^{+}$ECs after 4-8 weeks. We detected the presence of pericytes within single Confetti color clones (CFP, YFP or RFP; arrowheads in Figure $4 \mathrm{~J}-4 \mathrm{~L}$ ), and pericyte(s) always exhibited the same labeling color as the ECs, indicating that they share the same cellular origin. To be noted, no surrounding fibroblasts were labeled with the same color (Figure 4L), minimizing the possibility that these labeled pericytes were recruited from surrounding fibroblasts or generated by other Procr ${ }^{+}$stem cells. These data further demonstrate the de novo formation of pericytes from local Procr $^{+}$ECs.

Next we investigated the formation of pericytes using another Cre line, Cdh5-CreER [38]. Cdh5 is broadly expressed in ECs and its expression is restricted in ECs. We administered TAM to Cdh5-CreERT2;R2 $6^{m T m G /+} \mathrm{pu}$ bertal mice (5-week-old) and analyzed the labeled cells on mammary vasculature after 4 weeks of tracing (illustrated in Figure 4M). We observed the presence of GFP ${ }^{+}$ pericytes, indicating the EC origin of these pericytes (Figure $4 \mathrm{~N}$ ). Thus, with two independent Cre lines, our data demonstrate the de novo formation of pericytes from local ECs.

We analyzed vessel outgrowths in the previously described transplantation assays (Figures $1 \mathrm{~J}$ and $2 \mathrm{I}$ ), in which Procr ${ }^{+}$ECs isolated from Actin-GFP mice were injected, and also observed the presence of $\mathrm{GFP}^{+}$pericytes marked by NG2 expression (Supplementary information, Figure S5E). These data indicate that the $\mathrm{GFP}^{+}$pericytes are donor-derived from transplanted $\mathrm{Procr}^{+} \mathrm{ECs}\left(\mathrm{GFP}^{+}\right)$, not through recruitment from recipient tissues $\left(\mathrm{GFP}^{-}\right)$. In addition, we analyzed the formation of pericytes in cultured ECs. Both immunostaining and FACS analyses supported the idea that Procr ${ }^{+}$ECs can generate pericytes in vitro (Supplementary information, Figure S5F and S5G), whereas Procr ${ }^{-}$ECs cannot (Supplementary information, Figure $\mathrm{S} 5 \mathrm{H})$.

\section{Procr expression marks bipotent VESCs in multiple tis-} sues

Our in vitro culture, transplantation and lineage tracing experiments all suggest that Procr $^{+}$ECs are stem sells in mammary vasculature. Next we investigated the contribution of $\mathrm{Procr}^{+}$ECs in the vasculature of other adult tissues, including the skin and retina. Labeling was initiated in 5-week-old $\mathrm{PrOCr}^{\mathrm{CreERT2/+}} ; R 26^{m T_{m G /+}}$ mice, and abdominal skin and retinas were harvested at 2 days and 2 months post TAM induction (Figure 5A). In short-term ( 2 days) tracing, labeled cells were predominantly single ECs in the skin vasculature (Figure 5B), and we found no overlap of $\mathrm{GFP}^{+}$ECs with pericytes (Supplementary information, Figure S7B). After 2 months of tracing, clones with increased cell numbers were detected (Figure 5C and 5D). FACS analyses of the skin vasculature revealed that the percentage of $\mathrm{GFP}^{+}$ECs started from $3.4 \%$ at 2 days, and increased to $9.91 \%$ at 2 months and to $29.79 \%$ at 10 months (Figure 5E and Supplementary information, Figure S6C). Pericyte formation was also observed in the skin vasculature. At 2 months of tracing, $\mathrm{GFP}^{+}$pericytes were readily detected in $\mathrm{Procr}^{+}$EC-derived clones (Figure 5F and Supplementary information, Figure S7C). The frequency of pericyte(s) seen in GFP clones increased from $0 \%$ at 2 days to $21.73 \%$ at 2 months (Figure $5 \mathrm{G}$ ). Quantification of pericytes by FACS also demonstrated the overall percentage of labeled pericyte increased from $0 \%$ at 2 days, to $2.04 \%$ at 2 months and $9.09 \%$ to 10 months (Figure 5H and Supplementary information, Figure S6D).

Figure 4 Procr $^{+}$VESCs contribute to pericyte formation. (A) FACS analysis indicating that neither all ECs (grey) nor Procr ${ }^{+}$ ECs (red) express pericyte marker NG2. Pericyte was used as a positive control for NG2 expression (blue). (B) Whole-mount immunohistochemistry of mammary vasculature confirming that $\mathrm{Procr}^{+} \mathrm{ECs}$ (Procr ${ }^{+}, \mathrm{CD} 31^{+}$, arrow) do not overlap with pericytes (NG2 ${ }^{+}$, arrowhead). $n=206$ Procr $^{+}$cells scored. Scale bar, $50 \mu \mathrm{m}$. (C) FACS analysis of the stromal cell compartment indicating that pericytes (blue, marked by $\mathrm{NG}^{+}$or $\mathrm{Pdgfrb}^{+}$) and Procr-expressing fibroblasts (red) are distinct populations. (D) Whole-mount analysis of mammary vasculature at 2 days post TAM induction showing a GFP ${ }^{+}$EC (arrow) and GFP pericytes (arrowheads). Scale bar, $50 \mu \mathrm{m}$. (E, F) Whole-mount analysis of mammary vasculature at 2 months post TAM induction showing that a GFP ${ }^{+}$clone consisted of both ECs $\left(\mathrm{CD} 31^{+}\right)$and pericytes marked by NG2 (arrowheads in E) or Desmin (arrowheads in F). Scale bar, $50 \mu \mathrm{m}$. (G) Quantification of the percentage of clones containing GFP ${ }^{+}$pericytes. $16.1 \%$ of clones contained GFP ${ }^{+}$pericyte at 2 months of tracing, and the percentage increased to $32.1 \%$ at 10 months of tracing. Data are presented as mean \pm SD. Data were pooled from at least 3 mice for each tracing time point. (H, I) Quantification of GFP ${ }^{+}$ECs and $\mathrm{GFP}^{+}$pericytes in the mammary gland by FACS analysis, indicating the growing percentage of GFP ${ }^{+}$ECs $(\mathbf{H})$ and $\mathrm{GFP}^{+}$pericytes $(\mathrm{I})$ as tracing period prolongs. $(\mathrm{J}-\mathrm{L})$ Whole-mount confocal images indicating that CFP (J), YFP (K)

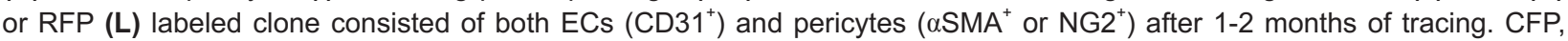
cyan fluorescent protein; RFP, red fluorescent protein; YFP, yellow fluorescent protein. Scale bar, $50 \mu \mathrm{m}$. (M) Illustration of lineage tracing strategy using Cdh5-CreERT2;R26 ${ }^{m T m G / t}$ line and experimental setup used. (N) Whole-mount confocal image

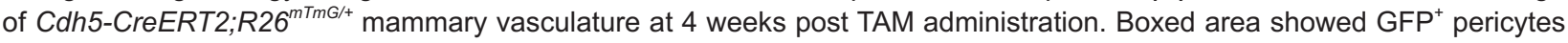
(arrowheads, $\mathrm{NG}^{+}$cells) within $\mathrm{GFP}^{+}$endothelial clones. Scale bar, $50 \mathrm{~mm}$. 

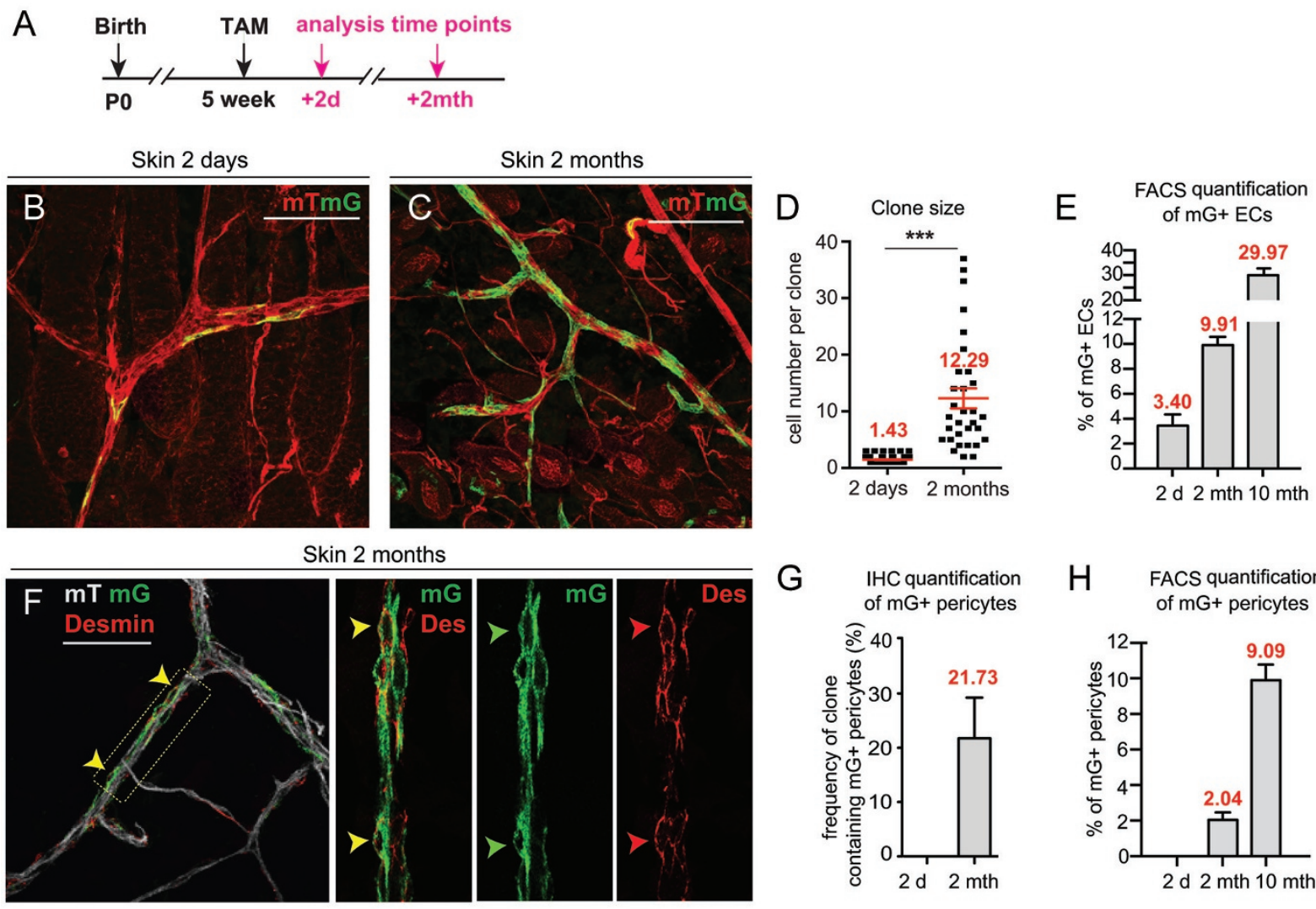

G

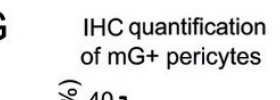

$\mathrm{H} \quad \begin{gathered}\text { FACS quantification } \\ \text { of } \mathrm{mG}+\text { pericytes }\end{gathered}$
H
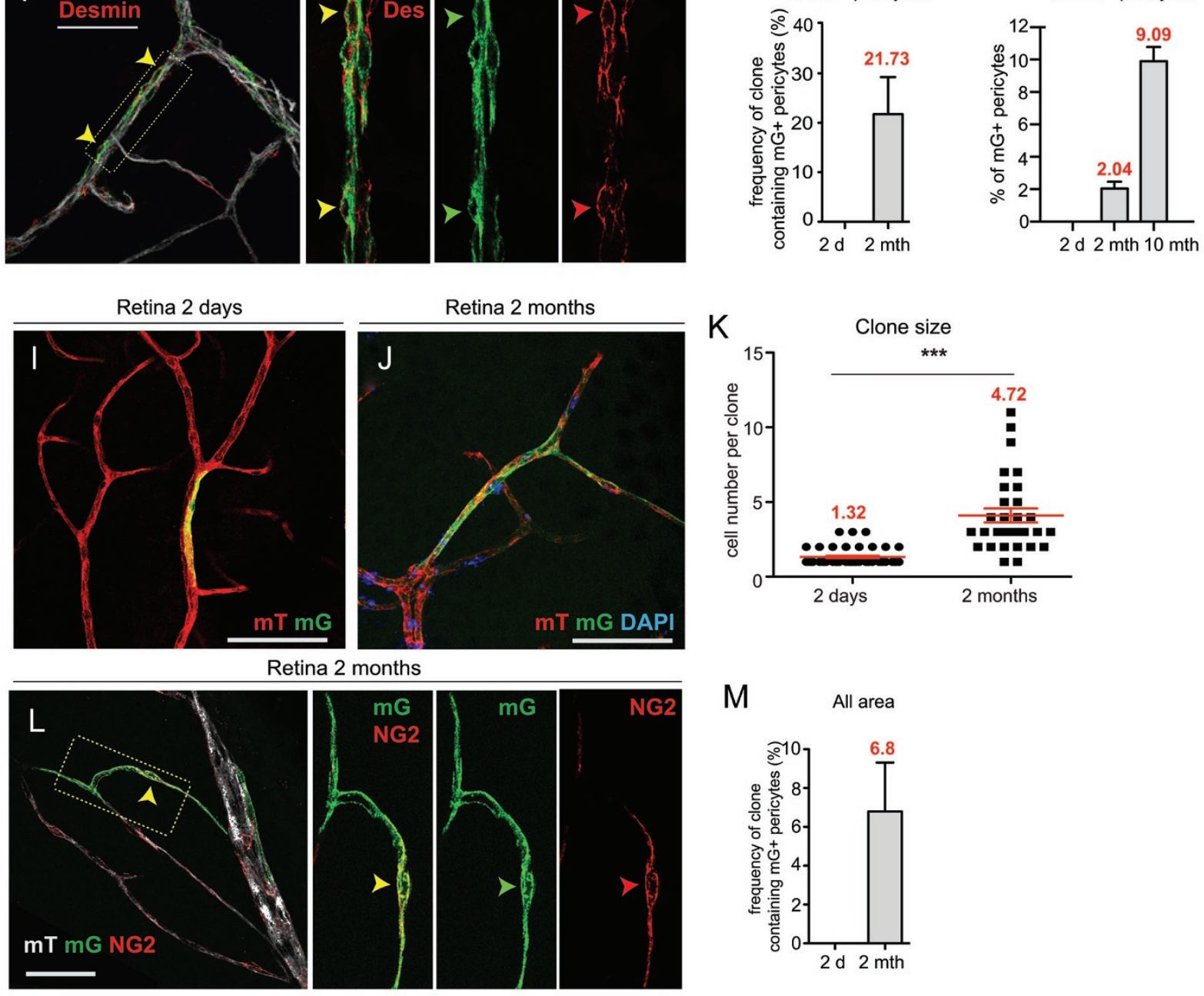

NG2

M

All area

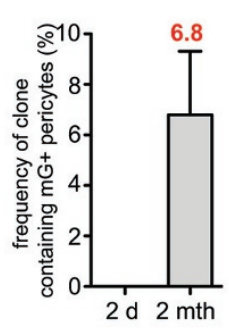


In retinal vasculature short-term tracing experiments, labeled cells were also predominantly single ECs in the retina (Figure 5I), and no overlap of $\mathrm{GFP}^{+}$ECs with pericytes was observed (Supplementary information, Figure S7D). After 2 months of tracing, clone sizes expanded (Figure $5 \mathrm{~J}$ and $5 \mathrm{~K}$ ), and $\mathrm{GFP}^{+}$pericytes appeared (Figure $5 \mathrm{~L}$ and Supplementary information, Figure S7E and S7F). Laminin staining confirmed that the $\mathrm{GFP}^{+}$pericytes and the $\mathrm{GFP}^{+}$ECs share the same basement membrane (Supplementary information, Figure S7G). The frequency of pericyte(s) seen in GFP clones increased from $0 \%$ at 2 days to $6.8 \%$ at 2 months (Figure 5M). Notably, the clones in the retina (Figure $5 \mathrm{~K}$ ) were smaller in size compared to the ones in the skin (Figure 5D), consistent with the notion of a slower turnover rate of the retina compared with the skin. Together, these results suggest that Procr ${ }^{+}$ECs act as stem cells that can generate both ECs and pericytes, and this could be a shared cellular mechanism in vasculatures of many organs.

\section{Procr $^{+}$VESCs are crucial for blood vessel development and regeneration}

To evaluate the functional importance of $\mathrm{Procr}^{+}$ VESCs during vascular development, we performed targeted ablation of these cells in the developing retina. Retinal vasculature develops postnatally in a tightly regulated temporal and spatial pattern that can readily be observed. The superficial vascular vessels develop immediately after birth by radial outgrowth of the vessels from the centre of the optic disc to the periphery, reaching the retinal edges at approximately postnatal day 8 , covering the superficial layer of the retina by forming a 2-D vascular structure [39]. We generated a Procr $^{\text {CreERT2/+ }} ; R 26^{\text {DTA/+ }}$ strain to conditionally express diphtheria toxin (DTA) in Procr $^{+}$cells. This allowed us to determine the requirement for Procr $^{+}$cells in retinal development. TAM was administered on day 1.5 and the retinas were harvested on day 6 (Figure 6A). We found that, compared to the control $\left(R 26^{D T A /+}\right)$, vascular development was severely delayed in $\mathrm{Procr}^{\mathrm{CreERT2/+}} ; \mathrm{R} 26^{\mathrm{DTA} /+}$ retinas, with vessel extension reaching only $\sim 50 \%$ of the entire retina diameter (Figure 6B and 6C). Of note, the phenotype of delayed development is likely because the efficiency of TAM-mediated recombination and cell ablation did not reach $100 \%$. As a result, the remaining Procr ${ }^{+}$VESCs escaped from the ablation and contributed to the partial development. These experiments suggest that Procr $^{+}$VESCs are important for normal retinal vascular development.

Next we investigated the regenerative potential of Procr ${ }^{+}$VESCs in vascular injury repair using a limb ischemia model. Hind limb ischemic injuries were induced in recipient mice by occlusion of the femoral artery, and mice were allowed to recover for 2 days after surgery. At that point, Procr ${ }^{+}$ECs or Procr ${ }^{-}$ECs were isolated from donor Actin-RFP mice and transplanted intramuscularly into the ischemic limbs. Laser Doppler analyses of blood flow were performed on day 2 and day 23 post surgery (Figure 6D). On day 23, mice treated with Procr $^{+}$ECs demonstrated significantly improved blood flow compared with the control animals that were treated with Procr ECs (Figure 6F-6H). Immunohistochemistry of the limb treated with Procr $^{+}$ECs revealed the formation of $\mathrm{RFP}^{+}$blood vessels, validating the contribution of the donor cells (Figure 6I). Isolectin staining indicated that those $\mathrm{RFP}^{+}$vessels are connected to the main circulation (Figure 6J). These results suggest that Procr ${ }^{+}$VESCs are effective in vascular restoration upon injury.

\section{Procr $^{+}$VESCs display angiogenic and EndMT molecular signatures}

To investigate the properties of $\mathrm{Procr}^{+}$VESCs, we performed RNA sequencing (RNA-seq) analysis on iso-

Figure 5 Procr $^{+}$VESCs contribute to both EC and pericyte formation in the skin and retina vasculature. (A) Experimental setup used in skin and retina vasculature lineage tracing. (B, C) Whole-mount confocal imaging of the abdominal skin vasculature showing individual GFP ${ }^{+}$cells at 2 days after TAM administration (B), and GFP ${ }^{+}$clones at 2 months after TAM induction (C). Scale bar, $100 \mu \mathrm{m}$. (D) Quantification of cell numbers per GFP ${ }^{+}$clone at each time point indicating the expansion of clone sizes. (E) FACS analysis indicating the increasing percentage of GFP ${ }^{+}$ECs. (F) At 2 months post TAM induction, the clones contained pericytes marked by Desmin (Des; arrowheads). Scale bar, $100 \mu \mathrm{m}$. (G) Quantification of the percentage of GFP clones that contained $\mathrm{GFP}^{+}$pericytes. No clones contained $\mathrm{GFP}^{+}$pericyte at 2 days of tracing, and the percentage increased to $21.73 \%$ at 2 months of tracing. (H) FACS quantification confirmed the increasing percentage of GFP ${ }^{+}$pericytes as tracing period prolongs. Data were pooled from at least 3 mice for each tracing time point and are presented as mean \pm SD. (I, J) Whole-mount confocal imaging of the retina vasculature showing individual GFP ${ }^{+}$cells at 2 days after TAM administration (I), and $\mathrm{GFP}^{+}$clones at 2 months after TAM induction (J). Scale bar, $50 \mu \mathrm{m}$. (K) Quantification of cell numbers per GFP ${ }^{+}$clone at each time point indicating the expansion of clone sizes. (L) At 2 months post TAM administration, the GFP ${ }^{+}$clones contained pericytes marked by NG2 (arrowhead). Scale bar, $50 \mu \mathrm{m}$. (M) Quantification of the percentage of GFP ${ }^{+}$clones that contained $\mathrm{GFP}^{+}$pericytes. When analyzed in all area, no clones contained $\mathrm{GFP}^{+}$pericyte at 2 days of tracing, and the percentage increased to $6.8 \%$ at 2 months of tracing. Data were pooled from at least 3 mice for each tracing time point and are presented as mean $\pm \mathrm{SD}$. 
A

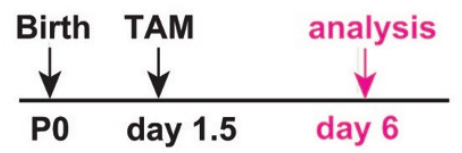

B

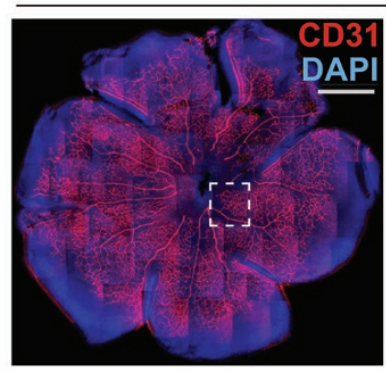

Day 6, Rosa-DTA (Ctrl)

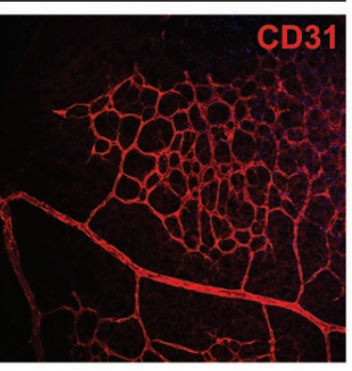

\section{Day 6, Procr-CreERT2;Rosa-DTA}

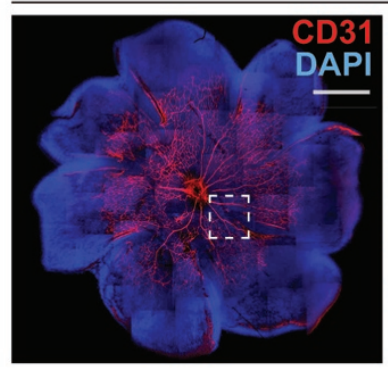

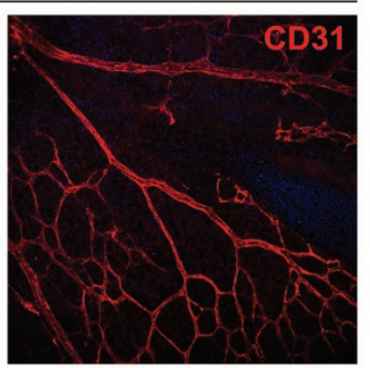

D

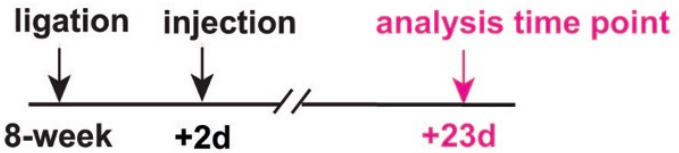

E

Day 2

F Ctrl (inject Procr- ECs), day 23

G Inject Procr+ ECs, day 23
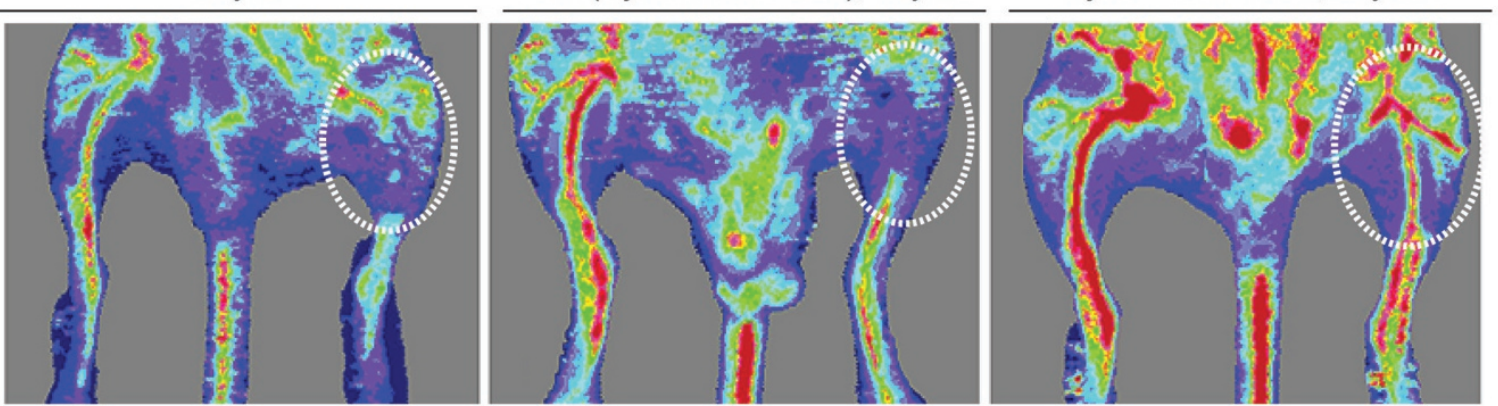

$\mathrm{H}$
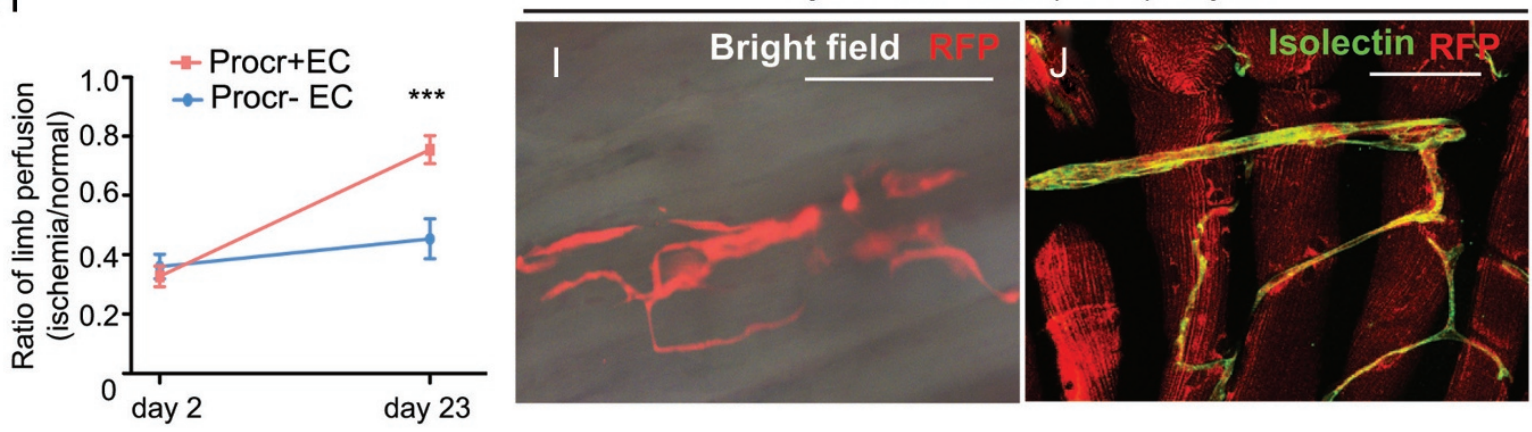

Figure 6 Procr $^{+}$VESCs are crucial for vascular development and regeneration. (A-C) Targeted ablation of Procr ${ }^{+}$cells delays retina blood vessel development. $\operatorname{Procr}^{\mathrm{CreERT2/+}} ; R 26^{D T A /+}$ mice and $R 26^{D T A /+}$ mice (Ctrl) were TAM administered at postnatal day 1.5 and retinas were harvested on day 6 as illustrated (A). The whole-mount view of entire retinal vascular occupancy as well as zoom-in view of vascular structure were visualized by CD31 whole-mount staining (B, C). Scale bar, $500 \mu \mathrm{m}$. (D-J) Mice were subjected to femoral artery ligation followed by intramuscular injection with Procr ${ }^{+}$ECs or Procr ${ }^{-}$ECs isolated from Actin-RFP mice on day 2. Representative laser-Doppler perfusion imaging of mice on day $2(\mathbf{E})$ and day $23(\mathbf{F}, \mathbf{G})$ after hind limb ischemia is shown. Quantification of blood flow recovery was calculated from $>3$ mice/group ( $n=3$ in Procr EC group, $n=5$ in Procr $^{+}$EC group). Data are presented as mean \pm SD. ${ }^{* *} P<0.01(\mathrm{H})$. Whole-mount microscopy image visualizing the formation of $\mathrm{RFP}^{+}$vessel in mice receiving Procr $^{+} \mathrm{EC}$ injection on day 23 (I). Confocal image confirming that these $\mathrm{RFP}^{+}$ vessels are connected to the systemic circulation as evidenced by isolectin staining (J). Scale bar, $50 \mu \mathrm{m}$. 
lated Procr $^{+}$and Procr ${ }^{-}$ECs from mammary vasculature. We found that Procr $^{+}$VESCs have higher expression of genes that have been implicated in angiogenesis and vascular development (Figure 7A), including surface receptors such as Vegfr1, Vegfr2, Tie1, Tie2, Notch1, and Notch3, potentially sensitizing VESCs' responsiveness to angiogenic stimuli. Many angiogenesis-related secreted molecules, transcription factors and adhesion molecules were also upregulated in $\mathrm{Procr}^{+}$VESCs. These observations were validated by qPCR experiments (Figure 7B7E). Interestingly, Procr $^{+}$VESCs exhibit characteristics of endothelial-to-mesenchymal transition (EndMT; Figure 7F). This is consistent with the postulation that a partial EndMT is required for initiating angiogenic sprouting [40]. In addition to the previously reported EndMT signatures [41], characteristic genes of epithelial-to-mesenchymal transition (EMT), e.g., Zeb1, Zeb2, Foxc2 and Vimentin were upregulated in Procr ${ }^{+}$VESCs (Figure 7F and $7 \mathrm{G}$ ). Consistently, transcription factors that are suppressed in EMT, e.g., Ovoll and Ovol2 were downregulated in Procr ${ }^{+}$VESCs (Figure 7G). To further validate these results, we performed immunostaining analysis of Zeb1, a central mediator of EMT [42]. We observed that Procr ${ }^{+}$VESCs indeed express Zeb1 (Figure 7H). Zeb1 is also expressed in perivascular cells as expected (Figure 7H). EndMT has been regarded as a subtype of EMT [43]. Analyses in Procr $^{+}$VESCs suggest that EndMT and EMT share more similarities in molecular signature than previously known.

\section{Discussion}

\section{Identifying bipotent VESCS}

Although much effort has been devoted to identifying the cellular origin of ECs [18, 25-28], the existence and the identity of VESCs remain elusive, due to the lack of in vivo evidence. Our study identifies Procr as a novel marker for VESCs through in vitro analyses and in vivo lineage tracing. Procr ${ }^{+}$VESCs have robust clonogenicity in culture and vigorous vessel formation capability in transplantation. Procr ${ }^{+}$VESCs actively contribute to the development and maintenance of homeostasis in postnatal angiogenesis in mammary, skin and retina vasculatures. In line with the robustness of tissue turnover, Procr ${ }^{+}$VESCs are more active in skin vasculature compared to the others, generating more descendent cells as evidenced by bigger clones in each assessed tracing period. In multiple adult tissues, Procr $^{+}$VESCs are bipotent, giving rise to new ECs and pericytes during development. This model argues against the current paradigm of a linear endothelial lineage, adding a subset of pericytes under the endothelial stem cell hierarchy. Tran- scriptome analysis indicates the prominent EndMT and EMT features of these VESCs. This is reminiscent of the EMT characteristic identified in Procr $^{+}$mammary epithelial stem cells [24], and may indicate a shared feature of these Procr-labeled adult stem cells.

\section{Pathological vs physiological EndMT}

EndMT is the process by which endothelial cells lose their cell-specific markers and morphology, and acquire a mesenchymal cell-like phenotype. EndMT has been observed in a range of pathological conditions. In cardiac fibrosis model, a lineage tracing study using Tie1-Cre mice (Tiel gene encodes a pan-endothelial cell marker) has demonstrated the significant contribution of vessel ECs to cardiac fibrotic tissue and scar formation through EndMT [44]. In vein grafting, a common revascularization surgery for patients suffering from coronary disease, the formation of aberrant aortic smooth muscle cell-like neointima through EndMT is associated with grafting failure [45]. EndMT has also been observed in the brain ECs in the context of cerebra cavernous malformation (CCM), a vascular dysplasia [41]. In heterotopic ossification disorders, ECs undergoing EndMT could acquire a mesenchymal stem cell-like phenotype, capable of differentiating into osteoblasts, chondrocytes and adipocytes [46]. In vitro, EndMT has also been documented in aortic ECs under the influence of Wnt7b signaling [47]. The above observations exemplify the potential of endothelial (stem) cells in pathological conditions and their plasticity in the in vitro setting. The true cell fate of endothelial (stem) cells, however, may only be manifested in vivo at certain windows during normal development. Our lineage tracing studies provide direct evidence that Procr $^{+}$ VESCs give rise to pericytes in normal development and homeostasis.

The current study not only identifies a novel source of pericytes, but also brings new insight into the cellular mechanism during pathological processes. For example, in fibrotic kidney disease, lineage tracing studies propose that pericytes were progenitors of myofibroblasts, which contribute to renal fibrosis [48, 49]. In light of our lineage tracing studies, it is possible that VESCs are the cells of origin of organ fibrosis under pathological conditions. The EndMT signatures that Procr $^{+}$VESCs carry could be advantageous for the mesenchymal fate switching during normal development (to become pericytes) or during pathological process (to further become fibroblasts).

\section{Diverse cellular origin of pericytes}

Pericytes displayed heterogeneity in their phenotype, distribution and origin [50-52]. The reported diverse 
A Procr+ endothelial cells RNA-seq Angiogenesis and vascular development

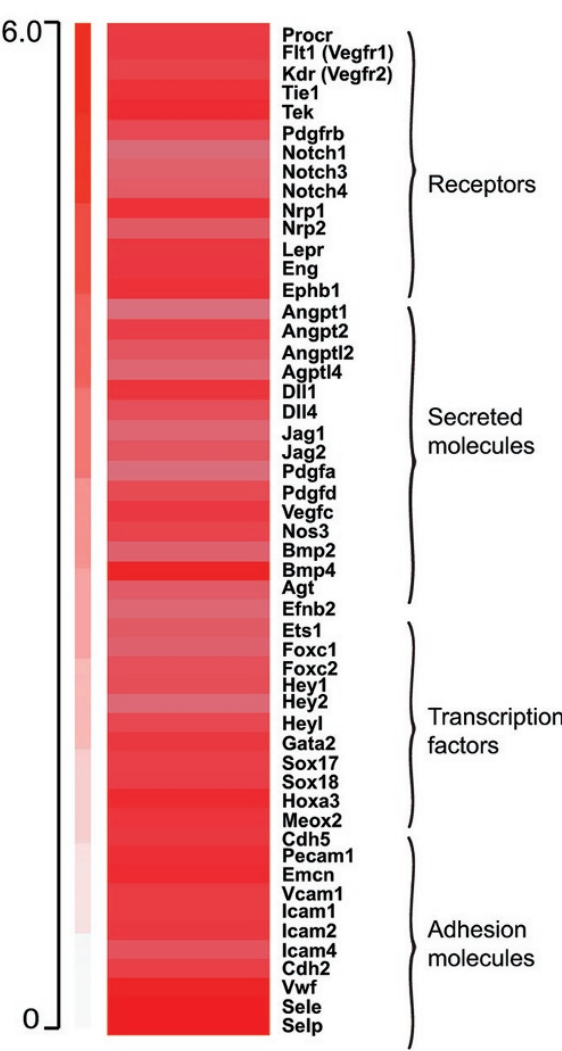

F

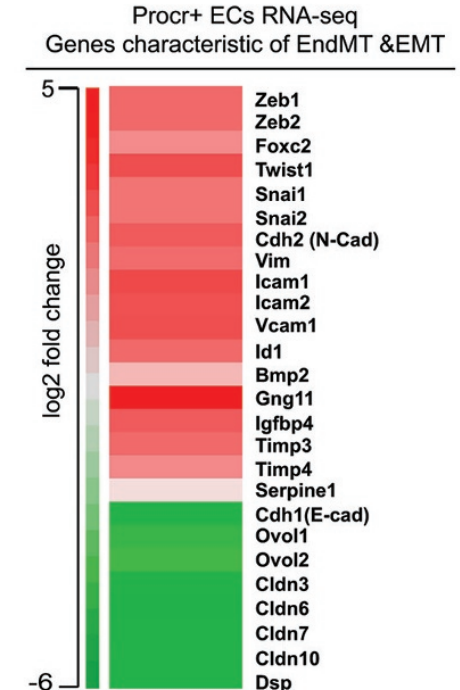

B

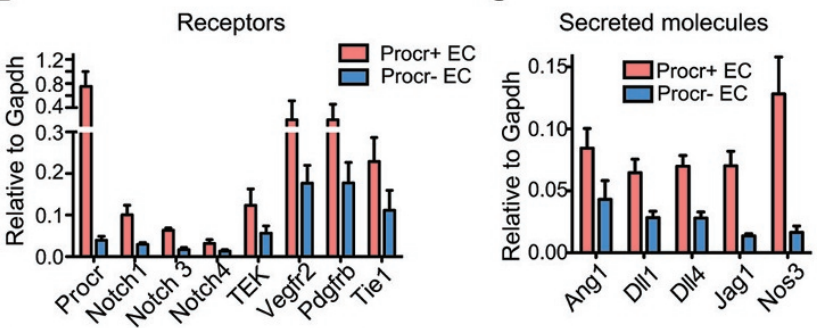

D

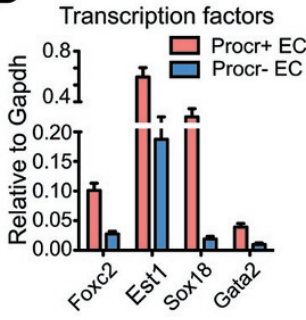

E

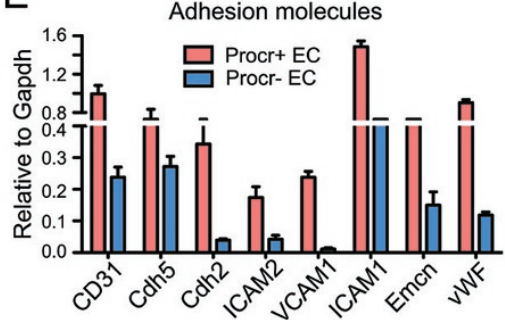

G
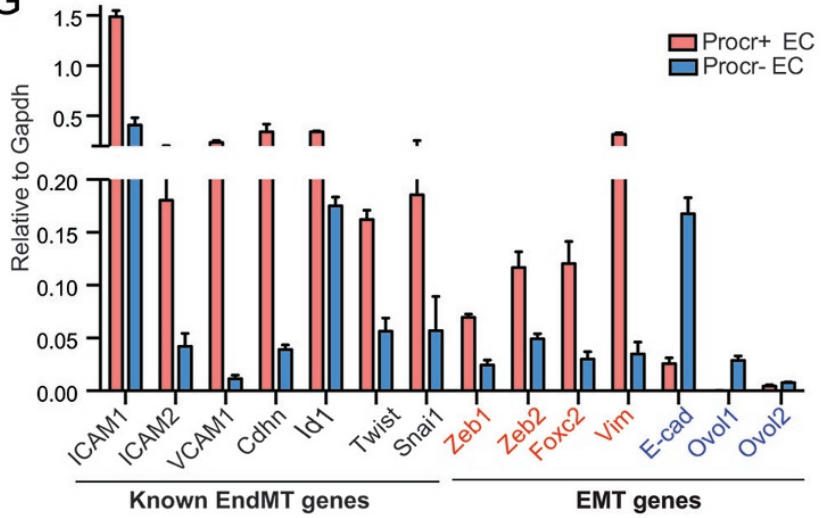

$\mathrm{H}$
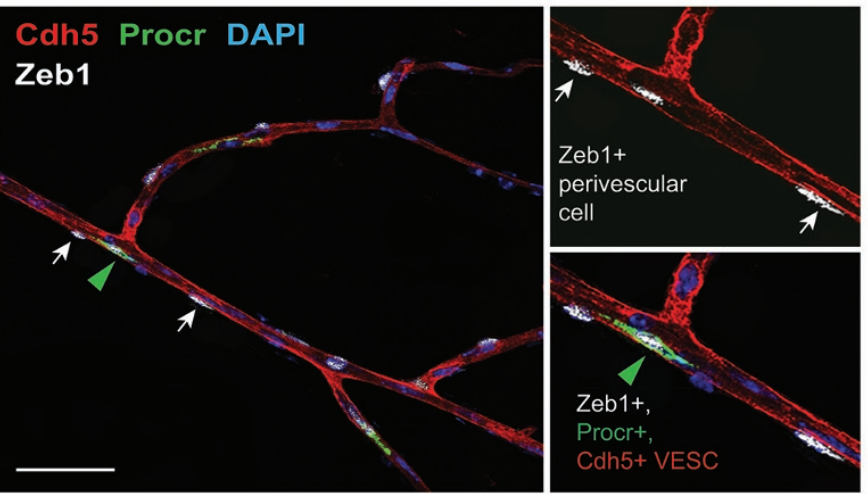

Figure 7 Procr $^{+}$VESCs have higher expression of angiogenic genes and display EndMT molecular signature. (A) RNA-seq analysis indicating upregulation of angiogenesis- and vascular development-related genes in Procr ${ }^{+} \mathrm{ECs}_{\mathrm{compared} \mathrm{to} \mathrm{Procr}}{ }^{-}$ ECs. (B-E) qPCR validation of RNA-seq results, including cell surface receptors (B), secreted molecules (C), transcription factors (D), and adhesion molecules (E) that are involved in angiogenesis and vascular development. (F) RNA-seq analysis indicating upregulation of characteristic genes of EndMT and EMT in Procr ${ }^{+}$VESCs. (G) qPCR validation of RNA-seq results showing higher expression of typical EndMT- and EMT-related genes in Procr ${ }^{+}$VESCs compared to Procr ${ }^{-}$ECs. (H) Wholemount confocal analysis of mammary vasculature showing Zeb1 staining on perivascular cells (arrows, Cdh5 ${ }^{-}$) and Zeb1 staining on Procr $^{+}$VESC (Cdh5 ${ }^{+}$, Procr ${ }^{-}$) as well (green arrowhead). Scale bar, $50 \mu \mathrm{m}$. 
cellular origin of pericytes is likely reflective of their heterogeneous nature. Early experiments using quail-chick chimera suggest that mural cells and smooth muscle cells in face and forebrain blood vessels are derived from neural crest [53]. Pericytes in the cephalic region are of neuroectodermal origin as demonstrated by lineage tracing using Sox10-iCreERT2 mice [54]. In the developing liver and lung, mesodermal lineages contribute to pericyte population as indicated by lineage tracing using $\mathrm{Me}$ sp1-Cre [7], a transiently expressed mesodermal marker in gastrulation, as well as using Wt1-Cre [9], which specifically marks mesothelial cells during embryonic stage [55]. Unlike conditional CreER of which the labeling efficiency is dependent on TAM induction efficacy, both of the mesodermal lineage studies have utilized Cre alleles which theoretically are capable of labeling all mesodermal cells, yet in both studies the labeled pericytes are only a portion of total pericytes in the organ, implying that there are other origins for pericytes.

Because of the reported distinct origins of pericytes with ECs, the model that pericytes are recruited to endothelial tubes, which is easy to comprehend, has been widely accepted. The experimental evidence of pericyte recruitment and their subsequent assembly with endothelial tubes is predominantly based on co-culture and co-transplantation assays. Co-culture of embryonic multipotent $10 \mathrm{~T} / 12$ cells with ECs has led to the differentiation and morphological change of those 10T/12 cells, exhibiting aSMA expression and vessel attachment [8]. More recently, pericyte recruitment is documented in tumor engraftment model. By grafting human osteosarcoma and fibrosarcoma cells into Desmin-lacZ mice, host-derived pericytes are observed in the growing tumors [11]. This has been presented as the major evidence of pericyte recruitment. However, ECs in those xenografts are likely also host derived, thereby an alternative explanation can also be that pericytes are derived from recruited ECs.

Accompanied with the recruitment model, it has been proposed that the arriving pericytes interact with local ECs to promote new basement membrane assembly. This is supported by the evidence that increased basement membrane deposition was observed in the co-culture of pericytes and ECs $[25,40]$. These interpretations reasonably depict the assembly of newly formed vessels at the tip. However, at established vessels during homeostasis, how the recruited pericytes or their progenitors penetrate through the basement membrane remains to be elucidated. Other mechanisms may exist for EC and pericyte assembly.

Our work provides direct evidence for the de novo formation of pericytes from local endothelial stem cells during vessel growth and renewal. These observations may reshape the understanding of blood vessel maturation and repair process.

\section{Mammary fat pat as a new tissue model to study angio- genesis}

Unlike other mammalian organs, the mammary gland develops mostly in the postnatal stage. At the onset of puberty, the mammary epithelium proliferates and extends to occupy the whole mammary fat pad. Convoying the establishing epithelium architecture is the robust remodeling of surrounding vascular structures. Thereby, the mammary gland offers an excellent model for the study of angiogenesis, in addition to other conventional tissue models, e.g., skin, retina and lung. Moreover, mammary fat pad is an ideal grafting site for assessing angiogenic potential of exogenous cells. It provides space, matrix and rich angiogenic stimuli from the growing mammary epithelium therein. It also allows the formed exogenous vessels to connect with the host circulation system, enabling a further functional evaluation and representing an advantage over subcutaneous transplantation. Last but not least, mammary fat pad that is outside the peritoneal cavity is very accessible; operation on it introduces limited distresses to the host.

\section{Conclusion}

Our study identifies Procr $^{+}$endothelial cells as bipotent VESCs in multiple vascular systems, and adds an additional local source to the diverse pericyte origin. Our study may provide more precise therapeutic targets to inhibit pathological angiogenesis and tumor growth, as well as provide new insight into the cellular contribution to fibrotic disorders.

\section{Materials and Methods}

\section{Experimental animals}

Rosa $26^{\text {mTmG/4}}$, Rosa2 $6^{\text {DTA/+ }}$ and Cdh5-CreER (Jackson Laboratories), Procr ${ }^{\text {CreERT2-IRES-tdTomato }}$ [24], Rosa26R-Confetti [56], Actin ${ }^{\text {GFP/+ }}$ $\operatorname{Actin}^{R F P / 4}, \mathrm{CD} 1, \mathrm{C} 57 \mathrm{BL} / 6 \mathrm{~J}$, and nude strains were used in this study. For lineage tracing experiments induced in pubertal and mature adult mice, animals received a single intraperitoneal injection of $4 \mathrm{mg}$ per $25 \mathrm{~g}$ body weight of TAM (Sigma-Aldrich) diluted in sunflower oil. A minimum of three mice were used for each tracing period. To obtain Procr ${ }^{\mathrm{CrEERT2} /+} ; R 26 R$-Confetti mice, R26R-Confetti mice were crossed with Procr ${ }^{\text {CreERT2-IRES-tdTomato }}$ mice. Random double heterozygous female mice between 5 and 8 weeks old received a three-course (on every second day for a period of 5 days) intraperitoneal injection of $4 \mathrm{mg}$ per $25 \mathrm{~g}$ body weight of TAM diluted in sunflower oil to induce activation of Cre recombinase, which in turn induced expression of one of the Confetti colors (nuclear GFP, membranous CFP, cytoplasmic YFP and RFP). To obtain 
Procr ${ }^{\text {CreERT2/+ }} ;$ Rosa $26^{\text {DTA/+ }}$ mice, Rosa $26^{\text {DTA/+ }}$ mice were crossed with Procr ${ }^{\text {CreERT2-IRES-tdTomato }}$ mice. Random double heterozygous pups received a double dose of intraperitoneal injection of $0.2 \mathrm{mg}$ TAM at postnatal day 1.5 to induce Cre recombinase-mediated cell ablation. Experimental procedures were approved by the Animal Care and Use Committee of Shanghai Institute of Biochemistry and Cell Biology, Chinese Academy of Sciences.

\section{Primary cell preparation and flow cytometry}

Primary cell preparation was conducted using previously reported protocol [24]. In brief, mammary glands of 8-week-old female virgin mice were harvested and single-cell suspension was obtained by Collagenase III (Worthington)-mediated digestion of minced tissue, followed by trypsin-EDTA and DNaseI (Sigma) sequential incubation before filtering through $70 \mu \mathrm{m}$ cell strainers. The following antibodies in 1:200 dilutions were used: rat anti-mouse CD16/CD32 (Mouse BD Fc Block ${ }^{\mathrm{TM}}$ BD PharMingen, clone \# 2.4G2), FITC-conjugated hematopoietic lineage cocktail (Biolegend, Cat\# 133302, cocktail includes antibody against CD3e, Ly-6G/Ly-6C, Gr-1, CD11b, CD45R/ B220, and Ter119), APC-conjugated CD31 (BD PharMingen, clone \# MEC 13.3), APC-conjugated CD140b (Biolegend, clone \# APB5), PE-Cy7-conjugated CD31 (eBiosciences, clone \# 390), APC- and PE-conjugated CD105 (eBioscience, clone \# MJ7/18), PE-Cy7-conjugated Scal (eBioscience, clone \# D7), FITC-conjugated c-Kit (BD Biosciences, clone \# 2B8) and Procr (eBioscience, clone \# eBio1560), APC-conjugated NG2 (Bioss, Cat\# bs4800R-A647), Streptavidin-v450 (BD PharMingen). Antibody incubation was performed on ice for $20 \mathrm{~min}$ in PBS with $5 \%$ fetal bovine serum (FBS; HyClone, Thermo Scientific). All analysis and sorting were performed using a FACSJazz (Becton Dickinson). The purity of sorted population was routinely checked and ensured to be $>95 \%$. Automated cell deposition unit in FACSJazz was used for single cell sorting of populations onto 96-multiwell plates, and the presence of the sorted cells was ensured using fluorescence microscopy.

\section{Cell culture and in vitro colony formation assay}

Freshly isolated endothelial cells were cultured on fibronectin (Sigma)-coated 24-well plates in IMDM medium containing 15\% FBS, 1\% BSA, $10^{-4} \mathrm{mM}$ 2-mercaptoethanol (Sigma), 1\% Glutamax, 1\% ITS-X (Insulin, transferrin and Selectin, Life Technologies), $100 \mathrm{ng} / \mathrm{ml} \mathrm{rmVEGFa} \mathrm{(R \& D} \mathrm{systems)} \mathrm{and} 50 \mathrm{ng} / \mathrm{ml} \mathrm{rmbFGF}$ (Life Technologies) at $37{ }^{\circ} \mathrm{C}$ in a humidified $5 \% \mathrm{CO}_{2}$ atmosphere (Esco Medical). Cells were passaged using 0.05\% trypsin when reaching $80 \%-90 \%$ confluence, fresh medium was supplied every 2-3 days depending on cell confluence. For in vitro endothelial cell colony assays, freshly isolated cells were plated in triplicate in $0.5 \mathrm{ml}$ of semi-solid methylcellulose (Stem Cell Technologies), supplemented with $100 \mathrm{ng} / \mathrm{ml} \mathrm{rmVEGFa}$. Colonies were scored after 7 days. Passage of primary colonies was achieved by single colony picking using a sterile pipette tip followed by digestion into single-cell suspension using $0.05 \%$ trypsin. Single cells were then re-plated into freshly supplemented methylcellulose medium. Colonies containing 10 or more cells were counted. To visualize the overall density of colonies in an entire well, the culture contents were fixed with 4\% PFA followed by wash and 30-min incubation with Crystal Violet dye and visualized using Leica MZ FLIII dissection microscope.

\section{Immunohistochemistry}

Mammary gland and abdominal skin whole-mount staining was performed as described previously [57] with adjustment to prolong tissue digestion period to better accommodate vasculature visualization. Retina dissection and whole-mount staining preparation was accomplished as previously demonstrated [58]. For immunohistochemistry on cell culture, cells were seeded onto fibronectin-coated 8-well chamber slides (Transwell, Lab-Tek) and allowed to reach confluence. Before antibody staining, cells in the chamber slides were washed with PBS and fixed in cold 4\% PFA for $15 \mathrm{~min}$, followed by 20 -min permeablization in $0.1 \%$ Triton $\mathrm{X}-100$ and 1-h blocking using 10\% of appropriate serum in PBS. Frozen sections were prepared by air-drying and fixation for 45 min in cold 4\% PFA, followed by the same permeablization and blocking procedures as cell culture. Tissue sections were incubated with primary antibodies at $4{ }^{\circ} \mathrm{C}$ overnight, followed by washes, incubation with secondary antibodies for $2 \mathrm{~h}$ at room temperature, and counterstained with DAPI (Life Technologies). Representative images were shown in the figures. The primary antibodies used in immunofluorescence were rat anti-mouse CD31 (BD Bioscience, clone \# MEC 13.3), rat anti-mouse Cdh5 (BD Biosciences, clone \# 11D4.1), rabbit anti-mouse NG2 (Millipore, Cat\# AB5320), rabbit anti-mouse Desmin (Millipore, Cat\# 04-585), rabbit anti-mouse Collagen Type IV (Millipore, Cat\# AB756P), mouse anti-mouse aSMA (Sigma-Aldrich, Cat\# A2547), rabbit anti-mouse laminin (Sigma-Aldrich, Cat\# L9393), rat anti-mouse Procr (eBioscience, clone \# eBio1560), hamster anti-mouse ICAM1 (BD Bioscience, clone \# 3E2), rat anti-mouse Endomucin (Santa Cruz, Cat\# sc53941 ), rabbit anti-mouse Keratin 14 (Covance), rabbit anti-mouse Pdgfrb (Cell Signaling Technology, Cat\# 3169), rabbit anti-mouse Phospho-eNOS Ser1177 (Cell Signaling Technology, Cat\# 9570), rabbit anti-mouse Zeb1 (Novus Biological, Cat\# NBP1-05987), and chicken anti-mouse GFP (Life Technologies, Cat\# A10262). The secondary antibodies used were donkey anti-rat Cy3, goat anti-rat Alexa Fluor 488, goat anti-rat Alexa Fluor 647, goat anti-mouse Alexa Fluor 647, goat anti-rabbit Alexa Fluor 647, donkey anti-goat Alexa Fluor 647 (Life Technologies). Fluorescence microscope images were captured using Zeiss AX10 and confocal images were captured using Leica DM6000 TCS/ SP8 laser confocal scanning microscope. All presented images were scanned as multiple layers at $1.0 \mu \mathrm{m}$ thickness each layer and z-stack processed (or $3 \mathrm{D}$ construction processed as per specified using Imaris 8.2.0 surface mode) to ensure correct co-localization of fluorescence signals.

\section{$A c-L D L$ uptake}

For analysis of Ac-LDL uptake of endothelial cells in culture, cells were incubated with $10 \mu \mathrm{g} / \mathrm{ml}$ Alexa Fluor 488-labeled AcLDL (Life Technologies) for $2 \mathrm{~h}$ at $37{ }^{\circ} \mathrm{C}$ with $5 \% \mathrm{CO}_{2}$ atmosphere, followed by fixation and immunostaining procedures.

\section{NO detection assays}

Passaged blood $1 \mathrm{in}^{-}, \mathrm{CD} 31^{+}, \mathrm{CD} 105^{+}, \mathrm{Procr}^{+} \mathrm{EC}$ cultures were used at $70 \%-80 \%$ of confluency. Cells were washed with PBS once and incubated with low-serum (2\% FBS) fresh medium supplemented with $10 \mu \mathrm{M}$ NO indicator DAF-FM Diacetate (Molecular Probes, Life Technologies) and processed according to the manufacturer's instructions. DAF-FM Diacetate excitation/ emission maxima is $495 / 505 \mathrm{~nm}$. Activation of eNOS was detected 
by immunofluorescence staining for the activation of Ser1117 site (rabbit anti Phospho-eNOS Ser1117, Cell Signaling), followed by secondary antibody staining using goat anti-rabbit Alexa Fluor 647 (Life Technologies). Fluorescence detection was visualized using Leica TCS/SP8 laser confocal microscope.

\section{Adhesion molecule expression}

Adhesion molecule expression was determined in a cell-based ELISA as previously described [29]. In brief, blood lin ${ }^{-}, \mathrm{CD} 31^{+}$, $\mathrm{CD} 105^{+}$, Procr $^{+}$ECs were FACS isolated and plated as monolayer culture in 24-multiwell plates till $70 \%-80 \%$ of confluency. Cultured cells either remained unstimulated or were stimulated with IL-1 $\beta(0.1-1 \mathrm{nM})$ for $24 \mathrm{~h}$ at $37^{\circ} \mathrm{C}$. Afterwards cells were washed with PBS and fixed with $2 \%$ PFA (pH 7.2). Cells were then processed for immunofluorescence staining with rat anti-mouse ICAM1 (eBioscience) followed by donkey anti-rat Cy3 (Life Technologies). Fluorescence detection was achieved using Leica TCS/SP8 laser confocal microscope.

Quantification of lineage-specific cells and the size of clones A minimum of three different mice were analyzed per tracing period. Dissociated single mammary cells were FACS analyzed for the composition of endothelial lineage and pericytes. For clonal analysis, a minimum of three experimental mice and a minimum of $100 \mathrm{GFP}^{+}$clones were analyzed per time point by whole-mount preparation. For each clone, the number of cells was scored in reference to $\mathrm{CD} 31$ or $\mathrm{Cdh} 5$ expression to assure their endothelial identity. For scoring of $\mathrm{GFP}^{+}$pericytes, cells were scored in reference to Pdgfrb, NG2, Desmine and $\alpha$ SMA expression or in relative location to laminin or Collagen IV basement membrane staining. $\mathrm{GFP}^{+}$pericytes were quantified by two approaches: first as "total clones", that is all counted $\mathrm{GFP}^{+}$clones regardless of the location and vessel types; second as "pericyte existing area", that is to include $\mathrm{GFP}^{+}$clones having pericyte located within $\left(\mathrm{GFP}^{+}\right)$and clones that have pericyte located nearby (1-3 cell distance to the clone edge, tdTomato ${ }^{+}$). For clone identification in $\operatorname{Procr}^{\mathrm{CreERT2/+}} ; R$ $26 R^{\text {Confetti } /+}$ mice, whole-mount tissue preparation was carried out and cells were scored in reference to the abovementioned endothelial or pericyte markers to assure their cellular identity. Fluorescence microscope images were captured using Leica DM6000 TCS/SP8 laser confocal scanning microscope. All presented images were scanned as multiple layers at $1.0 \mu \mathrm{m}$ thickness each layer and z-stack processed to ensure correct co-localization of fluorescence signals.

\section{EdU labeling}

In vivo EdU labeling was conducted by intraperitoneal injection of EdU mixture at $0.2 \mathrm{mg}$ per $10 \mathrm{~g}$ body weight into mice, followed by tissue harvest $2 \mathrm{~h}$ after incubation. Samples were subjected to Click-it chemistry (Invitrogen) prior to immunohistochemistry preparation.

\section{In vivo neovascularization using Matrigel}

8-week-old C57BL/6J mice were used as recipients. Freshly isolated cell populations were resuspended in $0.4 \mathrm{ml}$ Matrigel (Becton Dickinson) supplemented with 60 units/ml heparin (Sigma) and $150 \mathrm{ng} / \mathrm{ml} \mathrm{VEGF}$, and injected subcutaneously into the flank region. Matrigel plug was retrieved and analyzed 3-4 weeks later. Outgrowth were detected under fluorescent microscope (Zeiss) and categorized according to the total filled area of the Matrigel plug.

\section{Mammary fat pad transplantation and analysis}

Freshly isolated endothelial populations were resuspended in Matrigel mixture consisting of 50\% Matrigel, PBS with 20\% FBS, and $0.01 \%$ Trypan Blue (Sigma). Total volume of $15 \mu$ was injected into the fat pads of 3-week-old female nude mice. Mammary glands were harvested 4 weeks post transplantation. Donor-derived endothelial outgrowth and host vessel integration were detected after whole-mount preparation and images were captured by confocal microscope (Leica DM6000 TCS/SP8).

\section{Hind limb ischemia model and EC transplantation}

8-10-week-old nude mice were anaesthetised with intraperitoneal injection of Avertin. The proximal portions of the right femoral artery as well as the distal portion of the saphenous artery were double occluded and the obstructed portion of the artery was resected. Hind limb muscles were harvested 23 days after induction of ischemia. Controls were subjected to ischemia induction without EC transplantation. For EC transplantation, blood lin ${ }^{-}$, $\mathrm{CD}_{31}{ }^{+}, \mathrm{CD}_{105^{+}}$Procr $^{+}$or Procr ${ }^{-}$ECs were sorted from Actin $^{R F P /+}$ mice. The hind limb ischemia model was prepared and mice were allowed to rest for $48 \mathrm{~h}$ before local ischemic site muscular injection of EC populations.

\section{Laser Doppler blood flow analysis}

Hind limb blood flow was measured using a laser Doppler blood flow meter (LDBF; MoorLDI, Moor Instrument). LDBF analyses over the legs and paws were performed on postoperative day 2 and day 23. After scanning, sorted images were analyzed to quantify blood flow, and mean blood flow values of the ischemic and non-ischemic limb were calculated. To maintain consistency of data collection and prevent data variation due to ambient light and termperature, blood flow was expressed as the ratio of the left (non-ischemic) and right (ischemic) hind limb LDBF.

\section{RNA-seq and quantitative real-time PCR analysis}

Total RNAs from freshly isolated blood lin $^{-}, \mathrm{CD} 105^{+}, \mathrm{CD} 31^{+}$, Procr ${ }^{+}$and blood lin $^{-}, \mathrm{CD} 105^{+}, \mathrm{CD} 31^{+}$, Procr ${ }^{-}$ECs were extracted with RNAiso Plus (Takara) following the manufacturer's protocol. Total mRNA concentration was determined with NanoDrop ND-1000 and RNA-seq libraries were prepared according to the manufacturer's instruction (Illumina) followed by sequencing on Illumina HiSeq 2000, which was performed by BG-tech (Beijing Genomics Institute). In total, more than 35 million of $1 \times 100$ single reads for each sample were obtained and uniquely mapped to the mm9 mouse genome with $>75 \%$ mapping rate for both samples. Differential gene expression analysis was carried out and genes with significant alteration were extracted and further analyzed using DAVID Bioinformatics Resources 6.7. RNA-seq data can be viewed online under GEO accession number GSE69129. Expression of selected genes was validated and quantitative RTPCR was performed using TaqMan gene expression systems for mouse (Applied Biosystems).

\section{Statistical analysis}

Student's $t$-test was performed and the $P$-value was calculated in GraphPad PRISM 5.0 on data represented by histograms and dot plots, which consisted of results from at least three indepen- 
dent experiments unless specified otherwise. For all experiments with error bars, the standard deviation (SD) was calculated to indicate the variation within each experiment. When only two groups were compared, a two-sided Student's $t$-test was used. $P$ value of $<$ 0.05 was considered significant.

\section{Acknowledgments}

We are grateful to Drs Esther Verheyen and Chi-Chung Hui for critical reading of the manuscript and all lab members for helpful discussion. We thank Dr Bin Zhou for kindly providing the Cdh5CreER mouse. We thank Weimin Jiang, Liu Wei, Xiaorui Zhang and Shengkai Zuo for technical assistance. This work was supported by the National Natural Science Foundation of China (31530045 and 31371500 to YAZ, 31401245 to QCY), the Ministry of Science and Technology of China (2014CB964800 and 2012CB945000 to YAZ), the Chinese Academy of Sciences (XDA01010307 to YAZ) and Chinese Society of Cell Biology (Early Career Fellowship to QCY).

\section{Author Contributions}

YAZ conceived the study. QCY, WS and DW performed the experiments. YAZ and QCY analyzed the data and wrote the manuscript.

\section{Competing Financial Interests}

The authors declare no competing financial interests.

\section{References}

1 Diaz-Flores L, Gutierrez R, Varela H, Rancel N, Valladares F. Microvascular pericytes: a review of their morphological and functional characteristics. Histol Histopathol 1991; 6:269-286.

2 Hall CN, Reynell C, Gesslein B, et al. Capillary pericytes regulate cerebral blood flow in health and disease. Nature 2014; 508:55-60.

3 Pallone TL, Silldorff EP. Pericyte regulation of renal medullary blood flow. Exp Nephrol 2001; 9:165-170.

4 Hellström M, Gerhardt H, Kalén M, et al. Lack of pericytes leads to endothelial hyperplasia and abnormal vascular morphogenesis. J Cell Biol 2001; 153:543-553.

5 Lindahl P, Johansson BR, Leveen P, Betsholtz C. Pericyte loss and microaneurysm formation in PDGF-B-deficient mice. Science 1997; 277:242-245.

6 Simonavicius N, Ashenden M, van Weverwijk A, et al. Pericytes promote selective vessel regression to regulate vascular patterning. Blood 2012; 120:1516-1527.

7 Asahina K, Zhou B, Pu WT, Tsukamoto H. Septum transversum-derived mesothelium gives rise to hepatic stellate cells and perivascular mesenchymal cells in developing mouse liver. Hepatology 2011; 53:983-995.

8 Hirschi KK, Rohovsky SA, D'Amore PA. PDGF, TGF-beta, and heterotypic cell-cell interactions mediate endothelial cell-induced recruitment of 10T1/2 cells and their differentiation to a smooth muscle fate. J Cell Biol 1998; 141:805-814.

9 Que J, Wilm B, Hasegawa H, Wang F, Bader D, Hogan BL. Mesothelium contributes to vascular smooth muscle and mesenchyme during lung development. Proc Natl Acad Sci USA
2008; 105:16626-16630.

10 Yamashita J, Itoh H, Hirashima M, et al. Flk1-positive cells derived from embryonic stem cells serve as vascular progenitors. Nature 2000; 408:92-96.

11 Abramsson A, Berlin O, Papayan H, Paulin D, Shani M, Betsholtz C. Analysis of mural cell recruitment to tumor vessels. Circulation 2002; 105:112-117.

12 Wagers AJ, Weissman IL. Plasticity of adult stem cells. Cell 2004; 116:639-648.

13 Asahara T, Murohara T, Sullivan A, et al. Isolation of putative progenitor endothelial cells for angiogenesis. Science 1997; 275:964-967.

14 Bompais H, Chagraoui J, Canron X, et al. Human endothelial cells derived from circulating progenitors display specific functional properties compared with mature vessel wall endothelial cells. Blood 2004; 103:2577-2584.

15 Yoder MC, Mead LE, Prater D, et al. Redefining endothelial progenitor cells via clonal analysis and hematopoietic stem/ progenitor cell principals. Blood 2007; 109:1801-1809.

16 Rinkevich Y, Lindau P, Ueno H, Longaker MT, Weissman IL. Germ-layer and lineage-restricted stem/progenitors regenerate the mouse digit tip. Nature 2011; 476:409-413.

17 Wojakowski W, Tendera M, Michałowska A, et al. Mobilization of CD34/CXCR4+, CD34/CD117+, c-met+ stem cells, and mononuclear cells expressing early cardiac, muscle, and endothelial markers into peripheral blood in patients with acute myocardial infarction. Circulation 2004; 110:32133220 .

18 Fang S, Wei J, Pentinmikko N, Leinonen H, Salven P. Generation of functional blood vessels from a single c-kit + adult vascular endothelial stem cell. PLoS Biol 2012; 10:e1001407.

19 Snippert HJ, Clevers H. Tracking adult stem cells. EMBO Rep 2011; 12:113-122.

20 Bae JS, Yang L, Manithody C, Rezaie AR. The ligand occupancy of endothelial protein $\mathrm{C}$ receptor switches the protease-activated receptor 1-dependent signaling specificity of thrombin from a permeability-enhancing to a barrier-protective response in endothelial cells. Blood 2007; 110:39093916.

21 Fukudome K, Esmon CT. Identification, cloning, and regulation of a novel endothelial cell protein C/activated protein $\mathrm{C}$ receptor. J Biol Chem 1994; 269: 26486-26491.

22 Vetrano S, Ploplis VA, Sala E, et al. Unexpected role of anticoagulant protein $\mathrm{C}$ in controlling epithelial barrier integrity and intestinal inflammation. Proc Natl Acad Sci USA 2011; 108:19830-19835.

23 Balazs AB, Fabian AJ, Esmon CT, Mulligan RC. Endothelial protein $\mathrm{C}$ receptor (CD201) explicitly identifies hematopoietic stem cells in murine bone marrow. Blood 2006; 107:23172321.

24 Wang D, Cai C, Dong X, et al. Identification of multipotent mammary stem cells by protein $\mathrm{C}$ receptor expression. Nature 2015; 517:81-84.

$25 \mathrm{Hu}$ Y, Zhang Z, Torsney E, et al. Abundant progenitor cells in the adventitia contribute to atherosclerosis of vein grafts in ApoE-deficient mice. J Clin Invest 2004; 113:1258-1265.

26 Passman JN, Dong XR, Wu SP, et al. A sonic hedgehog signaling domain in the arterial adventitia supports resident Sca1+ smooth muscle progenitor cells. Proc Natl Acad Sci 
USA 2008; 105:9349-9354.

27 Sainz J, Al Haj Zen A, Caligiuri G, et al. Isolation of "side population" progenitor cells from healthy arteries of adult mice. Arterioscler Thromb Vasc Biol 2006; 26:281-286.

28 Naito H, Kidoya H, Sakimoto S, Wakabayashi T, Takakura $\mathrm{N}$. Identification and characterization of a resident vascular stem/progenitor cell population in preexisting blood vessels. EMBO J 2012; 31:842-855.

29 Patsch C, Challet-Meylan L, Thoma EC, et al. Generation of vascular endothelial and smooth muscle cells from human pluripotent stem cells. Nat Cell Biol 2015; 17:994-1003.

30 Zhang XQ, Takakura N, Oike Y, et al. Stromal cells expressing ephrin-B2 promote the growth and sprouting of ephrin-B2(+) endothelial cells. Blood 2001; 98:1028-1037.

31 Ding BS, Nolan DJ, Butler JM, et al. Inductive angiocrine signals from sinusoidal endothelium are required for liver regeneration. Nature 2010; 468:310-315.

32 Muzumdar MD, Tasic B, Miyamichi K, Li L, Luo L. A global double-fluorescent Cre reporter mouse. Genesis 2007; 45:593605.

33 Ozerdem U, Grako KA, Dahlin-Huppe K, Monosov E, Stallcup WB. NG2 proteoglycan is expressed exclusively by mural cells during vascular morphogenesis. Dev Dyn 2001; 222:218227.

34 Hellstrom M, Kalen M, Lindahl P, Abramsson A, Betsholtz C. Role of PDGF-B and PDGFR-beta in recruitment of vascular smooth muscle cells and pericytes during embryonic blood vessel formation in the mouse. Development 1999; 126:30473055.

35 Nehls V, Denzer K, Drenckhahn D. Pericyte involvement in capillary sprouting during angiogenesis in situ. Cell Tissue Res 1992; 270:469-474.

36 Nehls V, Drenckhahn D. The versatility of microvascular pericytes: from mesenchyme to smooth muscle? Histochemistry 1993; 99:1-12.

37 Snippert HJ, van der Flier LG, Sato T, et al. Intestinal crypt homeostasis results from neutral competition between symmetrically dividing Lgr5 stem cells. Cell 2010; 143:134-144.

38 Mahmoud M, Allinson KR, Zhai Z, et al. Pathogenesis of arteriovenous malformations in the absence of endoglin. Circ Res 2010; 106:1425-1433.

39 Stahl A, Connor KM, Sapieha P, et al. The mouse retina as an angiogenesis model. Invest Ophthalmol Vis Sci 2010; 51:2813-2826.

40 Welch-Reardon KM, Wu N, Hughes CC. A role for partial endothelial-mesenchymal transitions in angiogenesis? Arterioscler Thromb Vasc Biol 2015; 35:303-308.

41 Maddaluno L, Rudini N, Cuttano R, et al. EndMT contributes to the onset and progression of cerebral cavernous malformations. Nature 2013; 498:492-496.

42 Peinado H, Olmeda D, Cano A. Snail, Zeb and bHLH factors in tumour progression: an alliance against the epithelial phenotype? Nat Rev Cancer 2007; 7:415-428.

43 Piera-Velazquez S, Li Z, Jimenez SA. Role of endothelial-mesenchymal transition (EndoMT) in the pathogenesis of fibrotic disorders. Am J Pathol 2011; 179:1074-1080.

44 Zeisberg EM, Tarnavski O, Zeisberg M, et al. Endothelial-to-mesenchymal transition contributes to cardiac fibrosis. Nat Med 2007; 13:952-961.
45 Cooley BC, Nevado J, Mellad J, et al. TGF-beta signaling mediates endothelial-to-mesenchymal transition (EndMT) during vein graft remodeling. Sci Transl Med 2014; 6:227ra234.

46 Medici D, Shore EM, Lounev VY, Kaplan FS, Kalluri R, Olsen BR. Conversion of vascular endothelial cells into multipotent stem-like cells. Nat Med 2010; 16:1400-1406.

47 Cheng SL, Shao JS, Behrmann A, Krchma K, Towler DA. Dkk1 and MSX2-Wnt7b signaling reciprocally regulate the endothelial-mesenchymal transition in aortic endothelial cells. Arterioscler Thromb Vasc Biol 2013; 33:1679-1689.

48 Humphreys BD, Lin SL, Kobayashi A, et al. Fate tracing reveals the pericyte and not epithelial origin of myofibroblasts in kidney fibrosis. Am J Pathol 2010; 176:85-97.

49 Lin SL, Kisseleva T, Brenner DA, Duffield JS. Pericytes and perivascular fibroblasts are the primary source of collagen-producing cells in obstructive fibrosis of the kidney. Am J Pathol 2008; 173:1617-1627.

50 Armulik A, Genove G, Betsholtz C. Pericytes: developmental, physiological, and pathological perspectives, problems, and promises. Dev Cell 2011; 21:193-215.

51 Birbrair A, Zhang T, Wang ZM, Messi ML, Mintz A, Delbono O. Pericytes at the intersection between tissue regeneration and pathology. Clin Sci 2015; 128:81-93.

52 Sims DE. Diversity within pericytes. Clin Exp Pharmacol Physiol 2000; 27:842-846.

53 Etchevers HC, Vincent C, Le Douarin NM, Couly GF. The cephalic neural crest provides pericytes and smooth muscle cells to all blood vessels of the face and forebrain. Development 2001; 128:1059-1068.

54 Simon C, Lickert H, Gotz M, Dimou L. Sox10-iCreERT2: a mouse line to inducibly trace the neural crest and oligodendrocyte lineage. Genesis 2012; 50:506-515.

55 Raza A, Franklin MJ, Dudek AZ. Pericytes and vessel maturation during tumor angiogenesis and metastasis. Am J Hematol 2010; 85:593-598.

56 Sarkes D, Rameh LE. A novel HPLC-based approach makes possible the spatial characterization of cellular PtdIns5P and other phosphoinositides. Biochem J 2010; 428:375-384.

57 Rios AC, Fu NY, Lindeman GJ, Visvader JE. In situ identification of bipotent stem cells in the mammary gland. Nature 2014; 506:322-327.

58 Claybon A, Bishop AJ. Dissection of a mouse eye for a whole mount of the retinal pigment epithelium. J Vis Exp 2011 Feb 27. doi: $10.3791 / 2563$

(Supplementary information is linked to the online version of the paper on the Cell Research website.)

(c) (i) (s) $\Theta$ This work is licensed under a Creative Commons Attribution-NonCommercial-NoDerivs 4.0 Unported License. The images or other third party material in this article are included in the article's Creative Commons license, unless indicated otherwise in the credit line; if the material is not included under the Creative Commons license, users will need to obtain permission from the license holder to reproduce the material. To view a copy of this license, visit http://creativecommons.org/licenses/by-nc-nd/4.0/

(C) The Author(s) 2016 